\title{
A note on the Landauer principle in quantum statistical mechanics
}

\author{
Vojkan Jakšić ${ }^{1}$ and Claude-Alain Pillet ${ }^{2}$ \\ ${ }^{1}$ Department of Mathematics and Statistics, McGill University, \\ 805 Sherbrooke Street West, Montreal, QC, H3A 2K6, Canada \\ ${ }^{2}$ Université de Toulon, CNRS, CPT, UMR 7332, 83957 La Garde, France \\ Aix-Marseille Université, CNRS, CPT, UMR 7332, 13288 Marseille, France \\ FRUMAM
}

\begin{abstract}
The Landauer principle asserts that the energy cost of erasure of one bit of information by the action of a thermal reservoir in equilibrium at temperature $T$ is never less than $k_{\mathrm{B}} T \log 2$. We discuss Landauer's principle for quantum statistical models describing a finite level quantum system $\mathcal{S}$ coupled to an infinitely extended thermal reservoir $\mathcal{R}$. Using Araki's perturbation theory of KMS states and the Avron-Elgart adiabatic theorem we prove, under a natural ergodicity assumption on the joint system $\mathcal{S}+\mathcal{R}$, that Landauer's bound saturates for adiabatically switched interactions. The recent work [ReWo] on the subject is discussed and compared.
\end{abstract}

\section{Introduction}

Consider a quantum system $\mathcal{S}$ described by a finite dimensional Hilbert space $\mathcal{H}_{\mathcal{S}}$. Initially, $\mathcal{S}$ is in a state described by a density matrix $\rho_{\mathrm{i}}$. Let $\rho_{\mathrm{f}}$ be another density matrix on $\mathcal{H}_{\mathcal{S}}$. The Landauer principle [La, Ma] sets a lower bound on the energetic cost of the state transformation $\rho_{\mathrm{i}} \rightarrow \rho_{\mathrm{f}}$ induced by the action of a reservoir $\mathcal{R}$ in thermal equilibrium at temperature $T$. The principle can be derived from the second law of thermodynamics, provided one accepts that the (Clausius) entropy of the system $\mathcal{S}$ in the state $\rho_{\mathrm{i} / \mathrm{f}}$ coincides with its von Neumann entropy

$$
S\left(\rho_{\mathrm{i} / \mathrm{f}}\right)=-k_{\mathrm{B}} \operatorname{tr}\left(\rho_{\mathrm{i} / \mathrm{f}} \log \rho_{\mathrm{i} / \mathrm{f}}\right) .
$$

Since this is only correct if both $\rho_{\mathrm{i} / \mathrm{f}}$ are equilibrium states, such a derivation puts severe limits on the domain of validity of the Landauer principle, in contrast to its supposed universality and experimental verifications [Ber].

The derivation goes as follows. The decrease in the entropy of the system $\mathcal{S}$ in the transition $\rho_{\mathrm{i}} \rightarrow \rho_{\mathrm{f}}$ is

$$
\Delta S=S\left(\rho_{\mathrm{i}}\right)-S\left(\rho_{\mathrm{f}}\right) .
$$

Let $\Delta \mathcal{Q}$ denote the increase in the energy of the reservoir $\mathcal{R}$ in the same process. Assuming that the joint system $\mathcal{S}+\mathcal{R}$ is isolated and that the reservoir $\mathcal{R}$ is large enough to remain in equilibrium at temperature $T$ during the whole process, the entropy of $\mathcal{R}$ increases by $\Delta S_{\mathcal{R}}=\Delta \mathcal{Q} / T$ and the entropy balance equation of the process (see $[\mathrm{dGM}]$ ) reads

$$
\Delta S+\sigma=\frac{\Delta \mathcal{Q}}{T}
$$

where $\sigma$ is the entropy produced by the process. The second law of thermodynamics stipulates that $\sigma \geq 0$, with equality iff the transition is the result of a reversible quasi-static process. Hence, the inequality

$$
\Delta \mathcal{Q} \geq T \Delta S
$$


holds for arbitrary processes, with equality being achieved by reversible quasi-static processes in which the change of the total entropy vanishes

$$
\Delta S_{\text {tot }}=-\Delta S+\Delta S_{\mathcal{R}}=0
$$

With $d=\operatorname{dim} \mathcal{H}_{\mathcal{S}}, S\left(\rho_{\mathrm{i}}\right)$ is maximal and equal to $k_{\mathrm{B}} \log d$ if $\rho_{\mathrm{i}}=\mathbb{1} / d$ is the chaotic state and $S\left(\rho_{\mathrm{f}}\right)$ is minimal and equal to 0 if $\rho_{\mathrm{f}}=|\psi\rangle\langle\psi|$ is a pure state. It follows that

$$
\Delta \mathcal{Q} \geq k_{\mathrm{B}} T \log d
$$

If in addition $d=2$, then $\Delta \mathcal{Q}$ is the energy cost of the erasure of the qubit of information stored in $\rho_{\mathrm{i}}$ and (1.1) reduces to the Landauer bound.

The defects of the above "derivation" of the Landauer principle are manifest. In spite of its importance, there are very few mathematically rigorous results concerning the derivation of the Landauer bound from the first principles of statistical mechanics.

In an interesting recent work, Reeb and Wolf [ReWo] point out that the lack of mathematically precise formulation and proof of the Landauer principle in the context of quantum statistical mechanics has led to a number of controversies in the literature regarding its nature and validity. To remedy this fact, in the same work they provide a derivation of the Landauer principle which we will discuss in the next section.

One of the values of the paper [ReWo] is that it has brought the Landauer principle to the attention of researchers in quantum statistical mechanics.

In this note we shall examine the Landauer principle in the context of recent developments in the mathematical theory of open quantum systems ${ }^{1}$ ([AS],[ASF1]-[ASF4], [AJPP1, AJPP2, BFS, DJ, DJP, dR, dRK, FM, FMU, FMSU, JOP1, JOP2, JOPP], [JP1]-[JP7], [MMS1, MMS2, Pi1, Pi2, Ru1, Ru2, TM])2, and compare the outcome with the results of [ReWo].

The paper is organized as follows. In Section 2 we will review the work [ReWo]. The entropy balance equation in quantum statistical mechanics and its implication regarding Landauer's principle are presented in Section 3. We discuss the Landauer principle for instantaneously switched interactions in Section 4 and for adiabatically switched interactions in Section 5. Section 6 is devoted to the discussion of the results presented in this note. The proofs are given in Section 7.

This note is similar in spirit to the recent work [JOPS]. It is an attempt to bring together two directions of research which seem largely unaware of each other, in the hope that they both may benefit from this connection.

Acknowledgments. The research of V.J. was partly supported by NSERC. C.A.P. is grateful to the Department of Mathematics and Statistics at McGill University for its warm hospitality.

\section{The Reeb-Wolf derivation}

Suppose that $\mathcal{R}$ is described by a finite dimensional Hilbert space $\mathcal{H}_{\mathcal{R}}$ (we shall call such reservoirs confined) and Hamiltonian $H_{\mathcal{R}}$. Initially, $\mathcal{R}$ is in thermal equilibrium at temperature $T$, and its state is described by the density matrix

$$
\nu_{\mathrm{i}}=\mathrm{e}^{-\beta H_{\mathcal{R}}} / Z,
$$

where $Z=\operatorname{tr}\left(\mathrm{e}^{-\beta H_{\mathcal{R}}}\right)$ and $\beta=1 / T$ (in the following, we shall set Boltzmann's constant $k_{\mathrm{B}}$ to 1 ). The Hilbert space of the coupled system $\mathcal{S}+\mathcal{R}$ is

$$
\mathcal{H}=\mathcal{H}_{\mathcal{S}} \otimes \mathcal{H}_{\mathcal{R}}
$$

\footnotetext{
${ }^{1}$ We shall discuss the Landauer principle only for microscopic Hamiltonian models describing coupled system $\mathcal{S}+\mathcal{R}$. Repeated interaction systems (see [BJM]) are an instructive and physically important class of models in quantum statistical mechanics that also allow for mathematically rigorous analysis of the Landauer principle. This analysis is presented in [Raq].

${ }^{2}$ This is by no means a comprehensive list of references. Some of the earlier works that motivated these developments are [AM, BM, LeSp, McL, Rob, PW1, Sp1, Zu1, Zu2].
} 
and its initial state has the product structure

$$
\omega_{\mathrm{i}}=\rho_{\mathrm{i}} \otimes \nu_{\mathrm{i}}
$$

In what follows $\operatorname{tr}_{\mathcal{S} / \mathcal{R}}$ denotes the partial trace over $\mathcal{H}_{\mathcal{S} / \mathcal{R}}$ and, whenever the meaning is clear within the context, we will denote operators of the form $A \otimes \mathbb{1}, \mathbb{1} \otimes A$ by $A$. The relative entropy of two positive linear maps $\zeta_{1}, \zeta_{2}$ is defined by

$$
S\left(\zeta_{1} \mid \zeta_{2}\right)=\operatorname{tr}\left(\zeta_{1}\left(\log \zeta_{1}-\log \zeta_{2}\right)\right)
$$

If $\operatorname{tr} \zeta_{1}=\operatorname{tr} \zeta_{2}$, then $S\left(\zeta_{1} \mid \zeta_{2}\right) \geq 0$ with equality iff $\zeta_{1}=\zeta_{2}$.

Let $U: \mathcal{H} \rightarrow \mathcal{H}$ be a unitary operator inducing the state transformation

$$
\omega_{U}=U \omega_{\mathrm{i}} U^{*}
$$

The transformed states of the subsystems $\mathcal{S}$ and $\mathcal{R}$ are given by

$$
\rho_{U}=\operatorname{tr}_{\mathcal{R}}\left(\omega_{U}\right), \quad \nu_{U}=\operatorname{tr}_{\mathcal{S}}\left(\omega_{U}\right) .
$$

In the literature, the relative entropy

$$
S\left(\omega_{U} \mid \rho_{U} \otimes \nu_{U}\right)=S\left(\rho_{U}\right)+S\left(\nu_{U}\right)-S\left(\omega_{U}\right)
$$

is sometimes called mutual information and the fact that it is non-negative yields the subadditivity of entropy. The decrease in entropy of $\mathcal{S}$ and the increase in energy of $\mathcal{R}$ in the transition process $\omega_{\mathrm{i}} \rightarrow \omega_{U}$ are respectively

$$
\Delta S=S\left(\rho_{\mathrm{i}}\right)-S\left(\rho_{U}\right), \quad \Delta \mathcal{Q}=\operatorname{tr}\left(\nu_{U} H_{\mathcal{R}}\right)-\operatorname{tr}\left(\nu_{\mathrm{i}} H_{\mathcal{R}}\right) .
$$

The unitarity of $U$ and the product structure of $\omega_{\mathrm{i}}$ imply

$$
S\left(\omega_{U}\right)=S\left(\omega_{\mathrm{i}}\right)=S\left(\rho_{\mathrm{i}}\right)+S\left(\nu_{\mathrm{i}}\right)
$$

and Eq. (2.2) yields

$$
S\left(\nu_{\mathrm{i}}\right)=\beta \operatorname{tr}\left(\nu_{\mathrm{i}} H_{\mathcal{R}}\right)+\log Z .
$$

It follows that

$$
\begin{aligned}
S\left(\omega_{U} \mid \rho_{U} \otimes \nu_{\mathrm{i}}\right) & =-S\left(\omega_{U}\right)-\operatorname{tr}\left(\omega_{U}\left(\log \rho_{U}+\log \nu_{\mathrm{i}}\right)\right) \\
& =-S\left(\rho_{\mathrm{i}}\right)-S\left(\nu_{\mathrm{i}}\right)-\operatorname{tr}\left(\rho_{U} \log \rho_{U}\right)-\operatorname{tr}\left(\nu_{U} \log \nu_{\mathrm{i}}\right) \\
& =-S\left(\rho_{\mathrm{i}}\right)-\beta \operatorname{tr}\left(\nu_{\mathrm{i}} H_{\mathcal{R}}\right)-\log Z+S\left(\rho_{U}\right)+\beta \operatorname{tr}\left(\nu_{U} H_{\mathcal{R}}\right)+\log Z
\end{aligned}
$$

and one arrives at the entropy balance equation

$$
\Delta S+\sigma=\beta \Delta \mathcal{Q}
$$

where the entropy production term is given by

$$
\sigma=S\left(\omega_{U} \mid \rho_{U} \otimes \nu_{\mathrm{i}}\right) \geq 0 .
$$

This leads to the Landauer bound

$$
\beta \Delta \mathcal{Q} \geq \Delta S .
$$

Note that (2.5) implies that $\sigma=0$ iff $\omega_{U}=\rho_{U} \otimes \nu_{\mathrm{i}}$. The last relation yelds $\nu_{U}=\nu_{\mathrm{i}}$ and hence $\Delta \mathcal{Q}=0$. Thus equality holds in (2.6) iff $\Delta \mathcal{Q}=\Delta S=0$. In this case, it further follows from the identities

$$
\operatorname{tr}\left(\rho_{\mathrm{i}}^{\alpha}\right) \operatorname{tr}\left(\nu_{\mathrm{i}}^{\alpha}\right)=\operatorname{tr}\left(\omega_{\mathrm{i}}^{\alpha}\right)=\operatorname{tr}\left(\omega_{U}^{\alpha}\right)=\operatorname{tr}\left(\rho_{U}^{\alpha}\right) \operatorname{tr}\left(\nu_{\mathrm{i}}^{\alpha}\right),
$$

that $\operatorname{tr}\left(\rho_{\mathrm{i}}^{\alpha}\right)=\operatorname{tr}\left(\rho_{U}^{\alpha}\right)$ holds for all $\alpha \in \mathbb{C}$. One easily concludes from this fact that $\rho_{\mathrm{i}}$ and $\rho_{U}$ are unitarily equivalent. 
The following additional points are discussed in [ReWo].

Remark 1. Given $\rho_{\mathrm{i}}, \beta, \mathcal{H}_{\mathcal{R}}$ and $H_{\mathcal{R}}$, there are many target states $\rho_{\mathrm{f}}$ for which there is no unitary $U$ such that $\rho_{U}=\rho_{\mathrm{f}}$. Let

$$
\ell=e_{\max }-e_{\min }
$$

where $e_{\max } / \min$ is the maximal/minimal eigenvalue of $H_{\mathcal{R}}$. Then, for any unitary $U$,

$$
\mathrm{e}^{-\ell \beta} \rho_{\mathrm{i}} \leq \rho_{U} \leq \mathrm{e}^{\ell \beta} \rho_{\mathrm{i}}
$$

This constrains the set of possible target states $\rho_{\mathrm{f}}$. To reach a given $\rho_{\mathrm{f}}$, either exactly or up to a prescribed small error, one may need to adjust $\mathcal{H}_{\mathcal{R}}, H_{\mathcal{R}}$, and $U$. The following example illustrates one trivial way in which $\rho_{\mathrm{f}}$ can always be reached.

Example 1. Let $\rho_{\mathrm{f}}>0$ be the target state. Set $\mathcal{H}_{\mathcal{R}}=\mathcal{H}_{\mathcal{S}}, \nu_{\mathrm{i}}=\rho_{\mathrm{f}}, H_{\mathcal{R}}=-\log \rho_{\mathrm{f}}$. In this example, $\beta=1$. Let $U$ be the flip map, $U(\phi \otimes \psi)=\psi \otimes \phi$. Then $\rho_{U}=\rho_{\mathrm{f}}, \nu_{U}=\rho_{\mathrm{i}}$, the entropy production is

$$
\sigma=S\left(\rho_{\mathrm{i}} \mid \rho_{\mathrm{f}}\right),
$$

and $\Delta \mathcal{Q}=\Delta S$ iff $\rho_{\mathrm{f}}=\rho_{\mathrm{i}}$.

Remark 2. It turns out that Inequality (2.6), as a lower bound of $\Delta \mathcal{Q}$ in terms of $\Delta S$, is not optimal. This can be seen as follows, starting with the standard bound (see for example Theorem 1.15 in [OP])

$$
S\left(\omega_{U} \mid \rho_{U} \otimes \nu_{\mathrm{i}}\right) \geq \frac{1}{2}\left\|\omega_{U}-\rho_{U} \otimes \nu_{\mathrm{i}}\right\|_{1}^{2},
$$

where $\|X\|_{1}=\operatorname{tr}|X|=\sup _{A \neq 0}|\operatorname{tr}(A X)| /\|A\|$ is the trace norm. With $e=\left(e_{\max }+e_{\min }\right) / 2$, we can estimate

$$
\left\|\omega_{U}-\rho_{U} \otimes \nu_{\mathrm{i}}\right\|_{1} \geq \frac{\left|\operatorname{tr}\left[\left(H_{\mathcal{R}}-e\right)\left(\omega_{U}-\rho_{U} \otimes \nu_{\mathrm{i}}\right)\right]\right|}{\left\|H_{\mathcal{R}}-e\right\|}=\frac{|\Delta \mathcal{Q}|}{\ell / 2},
$$

and so the entropy production (2.5) satisfies

$$
\sigma \geq 2\left(\frac{\Delta \mathcal{Q}}{\ell}\right)^{2}
$$

Combining (2.4) and (2.7) and solving the resulting quadratic inequality shows that the possible entropy changes are restricted by the constraint $\Delta S \leq S_{0}=\beta^{2} \ell^{2} / 8$ and that the corresponding energy cost satisfies the improved bound

$$
\beta \Delta \mathcal{Q} \geq\left(1+\frac{1-\sqrt{1-\Delta S / S_{0}}}{1+\sqrt{1-\Delta S / S_{0}}}\right) \Delta S .
$$

A part of the discussion in [ReWo] is devoted to the refinement and optimization of the estimate (2.6) in the spirit of the above argument.

Example 2. On physical grounds, one expects saturation of the Landauer bound for quasi-static reversible processes. The following toy example of [ReWo] illustrates this point. Let $\rho_{\mathrm{f}}>0$ be a given target state. Let $\mathbb{R} \ni t \mapsto \rho(t)$ be a twice continuously differentiable map with values in density matrices on $\mathcal{H}_{\mathcal{S}}$ such that $\rho(0)=\rho_{\mathrm{i}}, \rho(1)=\rho_{\mathrm{f}}$, and $\rho(t)>0$ for $\left.\left.t \in\right] 0,1\right]$. Given a positive integer $N$, set $\rho_{n}=\rho(n / N)$, $\mathcal{H}_{\mathcal{R}}=\bigotimes_{n=1}^{N} \mathcal{H}_{\mathcal{S}}, \nu_{\mathrm{i}}=\bigotimes_{n=1}^{N} \rho_{n}$. With $\beta=1$, it follows that $H_{\mathcal{R}}=-\sum_{n=1}^{N} \log \rho_{n}, \omega_{\mathrm{i}}=\bigotimes_{n=0}^{N} \rho_{n}$. Let $U: \mathcal{H} \rightarrow \mathcal{H}$ be defined by

$$
U\left(\psi_{0} \otimes \psi_{1} \otimes \cdots \otimes \psi_{N}\right)=\psi_{N} \otimes \psi_{0} \otimes \cdots \otimes \psi_{N-1} .
$$

Then $\rho_{U}=\rho_{\mathrm{f}}$ and

$$
\Delta \mathcal{Q}_{N}=\operatorname{tr}\left(\nu_{U} H_{\mathcal{R}}\right)-\operatorname{tr}\left(\nu_{\mathrm{i}} H_{\mathcal{R}}\right)=\sum_{n=1}^{N} \operatorname{tr}\left[\left(\rho_{n}-\rho_{n-1}\right) \log \rho_{n}\right] .
$$


The differentiability assumption allows us to rewrite the r.h.s. of the previous identity as a Riemann sum, leading to

$$
\lim _{N \rightarrow \infty} \Delta \mathcal{Q}_{N}=\int_{0}^{1} \operatorname{tr}(\dot{\rho}(t) \log \rho(t)) \mathrm{d} t=S\left(\rho_{\mathrm{i}}\right)-S\left(\rho_{\mathrm{f}}\right) .
$$

In this example the number of steps $N$ plays the role of an adiabatic parameter and the limit $N \rightarrow \infty$ leads to a quasi-static process with optimal Landauer bound.

Remark 3. In the Landauer erasing principle $\rho_{\mathrm{i}}=\mathbb{1} / d$ and $\rho_{\mathrm{f}}=|\psi\rangle\langle\psi|$. Pure target states are thermodynamically singular and cannot be reached by the action of a thermal reservoir at strictly positive temperature. It follows from Example 2 that for any $\epsilon>0$ one can find $\rho_{\mathrm{f}}^{\prime}, \mathcal{H}_{\mathcal{R}}, \nu_{\mathrm{i}}$, and $U$ such that $\left\|\rho_{\mathrm{f}}-\rho_{\mathrm{f}}^{\prime}\right\|_{1}<\epsilon$, $\rho_{U}=\rho_{\mathrm{f}}^{\prime}$, and that the energy cost of the transformation $\rho_{\mathrm{i}} \rightarrow \rho_{\mathrm{f}}^{\prime}$ satisfies $\beta \Delta \mathcal{Q} \geq \log d-\epsilon$. The last result can be refined by considering infinite dimensional $\mathcal{H}_{\mathcal{R}}$ 's and allowing for Hamiltonians $H_{\mathcal{R}}$ with formally infinite energy levels. An additional toy example discussed in Section 6 of [ReWo] illustrates this point.

With the exception of the toy example mentioned in Remark 3, the work [ReWo] is exclusively concerned with finite dimensional thermal reservoirs. The authors discuss several additional topics including possible extensions of the notion of Landauer processes. The paper contains valuable discussions and clarifications concerning the physics literature on the Landauer principle. In the final Section 7 of the paper, the authors list a number of open problems/conjectures, including the following, on which we will comment later:

Conjecture [ReWo]. Landauer's Principle can probably be formulated within the general statistical mechanical framework of $C^{*}$ and $W^{*}$ dynamical systems [BR2, PWI, Th] and an equality version akin to (2.4) can possibly be proven. Note that in this framework the mutual information can be written as a relative entropy and the heat flow as a derivation w.r.t. the dynamical semigroup.

We now turn to the discussion of the Landauer principle in the context of the existing mathematical theory of open quantum systems.

\section{The entropy balance equation}

We start with the following remark regarding the derivation of the previous section. Let $\eta=\mathbb{1} \otimes \nu_{\mathrm{i}}$. Then

$$
S\left(\rho_{\mathrm{i}}\right)-S\left(\rho_{U}\right)+\sigma=S\left(\omega_{U} \mid \eta\right)-S\left(\omega_{\mathrm{i}} \mid \eta\right),
$$

and (2.4) can be written as

$$
S\left(\omega_{U} \mid \eta\right)-S\left(\omega_{\mathrm{i}} \mid \eta\right)=\beta \Delta \mathcal{Q} .
$$

The relation (3.8) is a special case of the general entropy balance equation in quantum statistical mechanics. In the form (3.8) it goes back at least to Pusz and Woronowicz (see the Remark at the end of Section 2 in [PW1]) and was rediscovered in [JP3, JP7, Pi1], see also [LeSp, McL, O1, O2, OHI, Sp2, Ru2, TM, $\mathrm{Zu} 1, \mathrm{Zu}$ ] ] for related works on the subject. To describe (3.8) in full generality we assume that the reader is familiar with basic definitions and results of algebraic quantum statistical mechanics, and in particular with Araki's perturbation theory of KMS structure. This material is standard and can be found in the monographs [BR1, BR2]. A modern exposition of the algebraic background can be found in [BF, DJP, Pi2]. The interested reader should also consult the fundamental paper [HHW]. For definiteness we will work with $C^{*}$-dynamical systems. With only notational changes all our results and proofs easily extend to $W^{*}$ dynamical systems and we leave such generalizations to the reader.

In the algebraic framework, a quantum system is described by a $C^{*}$-dynamical system $(\mathcal{O}, \tau)$. $\mathcal{O}$ is a $C^{*}$-algebra, with a unit $\mathbb{1}$, and $\tau$ is a strongly continuous one-parameter group of $*$-automorphisms of $\mathcal{O}$. Elements of $\mathcal{O}$ are observables, and their time evolution, in the Heisenberg picture, is given by $\tau$. We denote by $\mathcal{O}^{\text {sa }}$ the set of self-adjoint elements of $\mathcal{O}$. A state of the system is a positive linear functional 
$\omega$ on $\mathcal{O}$ such that $\omega(\mathbb{1})=1$. It is $\tau$-invariant if $\omega \circ \tau^{t}=\omega$ for all $t \in \mathbb{R}$. A thermal equilibrium state at inverse temperature $\beta$ is a $(\tau, \beta)$-KMS state. Such states are $\tau$-invariant.

Given a state $\omega$, the GNS construction provides a Hilbert space $\mathcal{H}_{\omega}$, a $*$-morphism $\pi_{\omega}: \mathcal{O} \rightarrow \mathcal{B}\left(\mathcal{H}_{\omega}\right)^{3}$ and a unit vector $\Omega_{\omega} \in \mathcal{H}_{\omega}$ such that $\pi_{\omega}(\mathcal{O}) \Omega_{\omega}$ is dense in $\mathcal{H}_{\omega}$ and $\omega(A)=\left\langle\Omega_{\omega}, \pi_{\omega}(A) \Omega_{\omega}\right\rangle$ for all $A \in \mathcal{O}$. A state given by $\zeta(A)=\operatorname{tr}\left(\rho \pi_{\omega}(A)\right)$, where $\rho$ is a density matrix on $\mathcal{H}_{\omega}$, is said to be $\omega$-normal. We denote by $\mathcal{N}_{\omega}$ the set of all $\omega$-normal states on $\mathcal{O}$. The state $\omega$ is called ergodic for $(\mathcal{O}, \tau)$ if, for all states $\zeta \in \mathcal{N}_{\omega}$ and all $A \in \mathcal{O}$,

$$
\lim _{t \rightarrow \infty} \frac{1}{t} \int_{0}^{t} \zeta\left(\tau^{s}(A)\right) \mathrm{d} s=\omega(A)
$$

and mixing if

$$
\lim _{t \rightarrow \infty} \zeta\left(\tau^{t}(A)\right)=\omega(A) .
$$

If $\omega$ is a $\tau$-invariant state, then there is a unique self-adjoint operator $L_{\omega}$ on $\mathcal{H}_{\omega}$ such that $L_{\omega} \Omega_{\omega}=0$ and $\pi_{\omega}\left(\tau^{t}(A)\right)=\mathrm{e}^{\mathrm{i} t L_{\omega}} \pi_{\omega}(A) \mathrm{e}^{-\mathrm{i} t L_{\omega}}$ for all $t \in \mathbb{R}$ and $A \in \mathcal{O} . L_{\omega}$ is called the $\omega$-Liouvillean of the dynamical system $(\mathcal{O}, \tau)$. If $\omega$ is a $(\tau, \beta)$-KMS state, then $L_{\omega}$ is also the standard Liouvillean (see Section 7) of $(\mathcal{O}, \tau)$.

The reservoir $\mathcal{R}$ is described by a $C^{*}$-dynamical system $\left(\mathcal{O}_{\mathcal{R}}, \tau_{\mathcal{R}}, \nu_{\mathrm{i}}\right)$ in thermal equilibrium at inverse temperature $\beta>0$. We denote by $\delta_{\mathcal{R}}$ the generator of $\tau_{\mathcal{R}}, \tau_{\mathcal{R}}^{t}=\mathrm{e}^{t \delta_{\mathcal{R}}}$ and by $L_{\mathcal{R}}$ its standard Liouvillean. If the reservoir is confined, then $\mathcal{O}_{\mathcal{R}}=\mathcal{B}\left(\mathcal{H}_{\mathcal{R}}\right), \delta_{\mathcal{R}}(\cdot)=\mathrm{i}\left[H_{\mathcal{R}}, \cdot\right]$, and $\nu_{\mathrm{i}}=\mathrm{e}^{-\beta H_{\mathcal{R}}} / \operatorname{tr}\left(\mathrm{e}^{-\beta H_{\mathcal{R}}}\right)^{4}$. The GNS Hilbert space $\mathcal{H}_{\nu_{\mathrm{i}}}$ is $\mathcal{O}_{\mathcal{R}}$ equipped with the inner product $\langle X, Y\rangle=\operatorname{tr}\left(X^{*} Y\right)$, the morphism $\pi_{\nu_{\mathrm{i}}}$ is defined by $\pi_{\nu_{\mathrm{i}}}(A) X=A X, \Omega_{\nu_{\mathrm{i}}}=\nu_{\mathrm{i}}^{1 / 2}$ and $L_{\mathcal{R}} X=\left[H_{\mathcal{R}}, X\right]$. However, in the remainder of this note we shall be concerned with infinitely extended reservoirs.

The $C^{*}$-algebra of the system $\mathcal{S}$, described by the finite dimensional Hilbert space $\mathcal{H}_{\mathcal{S}}$, is $\mathcal{O}_{\mathcal{S}}=\mathcal{B}\left(\mathcal{H}_{\mathcal{S}}\right)$. The $C^{*}$-algebra of the joint system $\mathcal{S}+\mathcal{R}$ is

$$
\mathcal{O}=\mathcal{O}_{\mathcal{S}} \otimes \mathcal{O}_{\mathcal{R}}
$$

and its initial state is

$$
\omega_{\mathrm{i}}=\rho_{\mathrm{i}} \otimes \nu_{\mathrm{i}},
$$

where $\rho_{\mathrm{i}}$ denotes the initial state of $\mathcal{S}$. We continue with our notational convention of omitting tensored identity, hence $\delta_{\mathcal{R}}=\mathrm{Id} \otimes \delta_{\mathcal{R}}$, etc.

Let $S\left(\zeta_{1} \mid \zeta_{2}\right)$ be the relative entropy of two positive linear functionals $\zeta_{1}, \zeta_{2}$ on $\mathcal{O}$ [Ar2, Ar3], with the ordering convention of [BR2, Don, JP3, JP6, DJP] and the sign convention of [Ar2, Ar3, OP] (with these conventions the relative entropy of two density matrices is given by (2.3)). The basic properties of the relative entropy are most easily deduced from the Pusz-Woronowicz-Kosaki variational formula [Ko, PW2]

$$
S\left(\zeta_{1} \mid \zeta_{2}\right)=\sup \int_{0}^{\infty}\left[\frac{\zeta_{2}(\mathbb{1})}{1+t}-\zeta_{2}\left(y^{*}(t) y(t)\right)-\frac{1}{t} \zeta_{1}\left(x(t) x^{*}(t)\right)\right] \frac{\mathrm{d} t}{t},
$$

where the supremum is taken over all countably valued step functions $[0, \infty[\ni t \mapsto x(t) \in \mathcal{O}$ vanishing in a neighborhood of zero and satisfying $x(t)+y(t)=\mathbb{1}$. In particular, if $\zeta_{1}(\mathbb{1})=\zeta_{2}(\mathbb{1})$, then $S\left(\zeta_{1} \mid \zeta_{2}\right) \geq 0$ with equality iff $\zeta_{1}=\zeta_{2}$.

Any unitary element $U \in \mathcal{O}$ induces a $*$-automorphism

$$
A \mapsto \alpha_{U}(A)=U^{*} A U,
$$

and hence a state transformation $\omega \mapsto \omega \circ \alpha_{U}$. Set $\eta=\mathbb{1} \otimes \nu_{\mathrm{i}}$. With this setup the entropy balance equation of [JP3, JP7, Pi1, PW1] reads as:

\footnotetext{
${ }^{3}$ Throughout the paper $\mathcal{B}(\mathcal{H})$ denotes the usual $C^{*}$-algebra of all bounded operators on a Hilbert space $\mathcal{H}$.

${ }^{4}$ If $\operatorname{dim} \mathcal{H}<\infty$, we shall not distinguish between positive linear functionals on $\mathcal{B}(\mathcal{H})$ and positive elements of $\mathcal{B}(\mathcal{H})$. They are identified by $\zeta(A)=\operatorname{tr}(\zeta A)$.
} 
Theorem 3.1 Suppose that $U \in \operatorname{Dom}\left(\delta_{\mathcal{R}}\right)$. Then

$$
S\left(\omega \circ \alpha_{U} \mid \eta\right)=S\left(\omega_{\mathrm{i}} \mid \eta\right)-\mathrm{i} \beta \omega_{\mathrm{i}}\left(U^{*} \delta_{\mathcal{R}}(U)\right)
$$

Denote by $\rho_{U}$ and $\nu_{U}$ the restriction of the transformed state $\omega \circ \alpha_{U}$ to $\mathcal{O}_{\mathcal{S}}$ and $\mathcal{O}_{\mathcal{R}}$ (i.e., $\rho_{U}(A)=$ $\omega \circ \alpha_{U}(A \otimes \mathbb{1})$ and $\nu_{U}(B)=\omega \circ \alpha_{U}(\mathbb{1} \otimes B)$ for $A \in \mathcal{O}_{\mathcal{S}}$ and $\left.B \in \mathcal{O}_{\mathcal{R}}\right)$. If $\mathcal{R}$ is confined, then

$$
-\mathrm{i} \omega_{\mathrm{i}}\left(U^{*} \delta_{\mathcal{R}}(U)\right)=-\mathrm{i} \omega_{\mathrm{i}}\left(U^{*} \mathrm{i}\left[H_{\mathcal{R}}, U\right]\right)=\omega_{\mathrm{i}}\left(\alpha_{U}\left(H_{\mathcal{R}}\right)-H_{\mathcal{R}}\right)=\operatorname{tr}\left(\nu_{U} H_{\mathcal{R}}\right)-\operatorname{tr}\left(\nu_{\mathrm{i}} H_{\mathcal{R}}\right),
$$

and (3.9) reduces to (3.8).

For any states $\rho$ on $\mathcal{O}_{\mathcal{S}}$ and $\omega$ on $\mathcal{O}$, Araki's perturbation formula for the relative entropy [Ar1] (see also Proposition 6.2.32 in [BR2] and Appendix A of [Don]) gives

$$
S\left(\omega \mid \rho \otimes \nu_{\mathrm{i}}\right)=S(\omega \mid \eta)-\omega(\log \rho) .
$$

Setting $\omega=\omega_{\mathrm{i}}=\rho_{\mathrm{i}} \otimes \nu_{\mathrm{i}}$, this implies in particular that

$$
S\left(\rho_{\mathrm{i}}\right)=-S\left(\omega_{\mathrm{i}} \mid \eta\right)
$$

The entropy balance equation (3.9) allows for an analysis of Landauer's principle in the general setup of quantum statistical mechanics. The decrease in entropy of $\mathcal{S}$ and the increase in energy of $\mathcal{R}$ in the transition process $\omega_{\mathrm{i}} \rightarrow \omega \circ \alpha_{U}$ are

$$
\Delta S=S\left(\rho_{\mathrm{i}}\right)-S\left(\rho_{U}\right), \quad \Delta \mathcal{Q}=-\mathrm{i} \omega_{\mathrm{i}}\left(U^{*} \delta_{\mathcal{R}}(U)\right) .
$$

Writing (3.9) as

$$
\Delta S+\sigma=\beta \Delta \mathcal{Q}
$$

and taking (3.11) into account yields

$$
\sigma=S\left(\omega \circ \alpha_{U} \mid \eta\right)+S\left(\rho_{U}\right)
$$

Since $S\left(\rho_{U}\right)=-\omega \circ \alpha_{U}\left(\log \rho_{U}\right)$, Eq. (3.10) further gives

$$
\sigma=S\left(\omega \circ \alpha_{U} \mid \rho_{U} \otimes \nu_{\mathrm{i}}\right)
$$

and hence

$$
\sigma \geq 0
$$

with equality iff $\omega \circ \alpha_{U}=\rho_{U} \otimes \nu_{\mathrm{i}}$. This implies the Landauer bound

$$
\beta \Delta \mathcal{Q} \geq \Delta S
$$

for the state transformation induced by the inner $*$-automorphism $\alpha_{U}$. This also settles the conjecture of [ReWo] which has in fact been been known for many years.

The analysis of the saturation of the Landauer bound is more delicate than in the case of a confined reservoir. It relies on the spectral analysis of modular operators. We shall give one result in this direction.

Proposition 3.2 Assume that the point spectrum of the standard Liouvillean $L_{\mathcal{R}}$ is finite. Then $\Delta S=$ $\beta \Delta \mathcal{Q}$ if and only if $\Delta S=\Delta \mathcal{Q}=0$ in which case $\rho_{U}$ is unitarily equivalent to $\rho$ and $\nu_{U}=\nu_{\mathrm{i}}$

Remark. If $\mathcal{R}$ is confined, then the spectrum of $L_{\mathcal{R}}$ is discrete and finite so that the above proposition applies. It also applies to the physically important class of ergodic extended reservoirs. Indeed, it follows from Theorem 1.2 in [JP4] that 0 is the only eigenvalue of $L_{\mathcal{R}}$ if $\nu_{\mathrm{i}}$ is an ergodic state for $\left(\mathcal{O}_{\mathcal{R}}, \tau_{\mathcal{R}}\right)$. It is an interesting structural question to characterize all reservoir systems for which the conclusions of Proposition 3.2 holds. 
One can continue with the abstract analysis of the Landauer principle in the above framework. As in the finite dimensional case, the bound

$$
\sigma \geq \frac{1}{2}\left\|\omega \circ \alpha_{U}-\rho_{U} \otimes \nu_{\mathrm{i}}\right\|^{2}
$$

follows from Eq. (3.13), the norm on the right hand side being dual to the $C^{*}$-norm of $\mathcal{O}$. Since

$$
\begin{aligned}
& \left\|\nu_{U}-\nu_{\mathrm{i}}\right\|=\sup _{0 \neq A \in \mathcal{O}_{\mathcal{R}}} \frac{\left|\omega \circ \alpha_{U}(\mathbb{1} \otimes A)-\rho_{U} \otimes \nu_{\mathrm{i}}(\mathbb{1} \otimes A)\right|}{\|\mathbb{1} \otimes A\|} \\
& \leq \sup _{0 \neq A \in \mathcal{O}} \frac{\left|\omega \circ \alpha_{U}(A)-\rho_{U} \otimes \nu_{i}(A)\right|}{\|A\|}=\left\|\omega \circ \alpha_{U}-\rho_{U} \otimes \nu_{\mathrm{i}}\right\|,
\end{aligned}
$$

(3.14) gives

$$
\sigma \geq \frac{1}{2}\left\|\nu_{U}-\nu_{\mathrm{i}}\right\|^{2}
$$

Suppose that $\rho_{\mathrm{i}}>0$ and let $\rho_{\mathrm{f}}>0$ be a target state. Set $\omega_{\mathrm{f}}=\rho_{\mathrm{f}} \otimes \nu_{\mathrm{i}}$. Another application of Araki's perturbation formula gives

$$
\sigma=S\left(\omega \circ \alpha_{U} \mid \rho_{U} \otimes \nu_{\mathrm{i}}\right)=S\left(\omega \circ \alpha_{U} \mid \omega_{\mathrm{f}}\right)-S\left(\rho_{U} \mid \rho_{\mathrm{f}}\right)
$$

Assume that there exists a sequence $U_{n}$ of unitary elements of $\mathcal{O}$ such that $U_{n} \in \operatorname{Dom}\left(\delta_{\mathcal{R}}\right)$ and

$$
\lim _{n \rightarrow \infty} \omega \circ \alpha_{U_{n}}(A)=\omega_{\mathrm{f}}(A)
$$

for all $A \in \mathcal{O}$. Since this implies that $\rho_{U_{n}} \rightarrow \rho_{\mathrm{f}}$, it follows from the entropy balance relation (3.12) that

$$
\liminf _{n \rightarrow \infty} \beta \Delta \mathcal{Q}_{n}=\liminf _{n \rightarrow \infty} \sigma_{n}+S\left(\rho_{\mathrm{i}}\right)-S\left(\rho_{\mathrm{f}}\right) \geq S\left(\rho_{\mathrm{i}}\right)-S\left(\rho_{\mathrm{f}}\right) .
$$

Moreover,

$$
\lim _{n \rightarrow \infty} \beta \Delta \mathcal{Q}_{n}=S\left(\rho_{\mathrm{i}}\right)-S\left(\rho_{\mathrm{f}}\right)
$$

if and only if

$$
\lim _{n \rightarrow \infty} \sigma_{n}=0
$$

which, by (3.16), is equivalent to

$$
\lim _{n \rightarrow \infty} S\left(\omega \circ \alpha_{U_{n}} \mid \omega_{\mathrm{f}}\right)=0
$$

The relation (3.18) quantifies the notion of quasi-static transition process. If (3.18) holds, then Inequality (3.15) implies

$$
\lim _{n \rightarrow \infty}\left\|\omega \circ \alpha_{U_{n}}-\omega_{\mathrm{f}}\right\|=0 .
$$

On the other hand, the norm convergence (3.19) does not imply (3.18). Sufficient conditions for (3.18) are discussed in the foundational papers [Ar1, Ar2]. For example, if in addition to (3.19) there is $\lambda>0$ such that

$$
\lambda \omega \circ \alpha_{U_{n}} \geq \omega_{\mathrm{f}}
$$

for all $n$, then (3.18) holds. A sufficient condition for (3.20) is that

$$
\sup _{n}\left\|\mathrm{e}^{-\mathrm{i} \delta_{\mathcal{R}} / 2 \beta}\left(U_{n}\right)\right\|<\infty \text {. }
$$

Remark. If the quantum dynamical system $\left(\mathcal{O}_{\mathcal{R}}, \tau_{\mathcal{R}}, \nu_{\mathrm{i}}\right)$ describes an infinitely extended reservoir in thermal equilibrium at positive temperature and in a pure phase, then on physical grounds it is natural to assume that the enveloping von Neumann algebra $\pi_{\nu_{\mathrm{i}}}\left(\mathcal{O}_{\mathcal{R}}\right)^{\prime \prime}$ is an injective factor of type $\mathrm{III}_{1}$ (see, e.g., [Ar4, ArW, Hu]). In this case, it is a simple consequence of a result of Connes and Størmer (Theorem 4 
in [CSt]) and Kaplansky's density theorem (Corollary 5.3.7 in [KR]) that there is a sequence of unitaries $U_{n} \in \operatorname{Dom}\left(\delta_{\mathcal{R}}\right)$ such that (3.19) and hence (3.17) holds.

Although one can go quite far by continuing the above structural analysis of the Landauer principle, we shall not pursue this direction further. Instead, we shall focus on physically relevant realizations of $\alpha_{U}$ 's by considering the dynamics of the coupled system $\mathcal{S}+\mathcal{R}$ and we shall analyze the Landauer principle in this context. Non-trivial dynamics are characterized by interactions that allow energy/entropy flow between $\mathcal{S}$ and $\mathcal{R}$. We shall distinguish between instantaneously and adiabatically switched interactions.

\section{Instantaneously switched interactions}

\subsection{Setup}

For $K \in \mathcal{O}^{\text {sa }}$, the $*$-derivation

$$
\delta_{K}=\delta_{\mathcal{R}}+\mathrm{i}[K, \cdot]
$$

generates a strongly continuous group $\tau_{K}^{t}=\mathrm{e}^{t \delta_{K}}$ of $*$-automorphisms of $\mathcal{O}$. Self-adjoint elements of $\mathcal{O}$ are called local perturbations and the group $\tau_{K}$ is the local perturbation of $\tau_{\mathcal{R}}$ induced by $K$. For example, if $H_{\mathcal{S}}$ is the Hamiltonian of $\mathcal{S}$ and $V$ describes the interaction of $\mathcal{S}$ with $\mathcal{R}$, then the dynamics of the interacting system $\mathcal{S}+\mathcal{R}$ is given by $\tau_{K}$, with $K=H_{\mathcal{S}}+V$. In this section, we investigate the Landauer principle for the dynamical system $\left(\mathcal{O}, \tau_{K}\right)$.

The interacting dynamics can be expressed as

$$
\tau_{K}^{t}(A)=\tau_{\mathcal{R}}^{t}\left(U_{K}^{*}(t) A U_{K}(t)\right),
$$

where the interaction picture propagator $U_{K}(t)$ is a family of unitary elements of $\mathcal{O}$ satisfying

$$
\mathrm{i} \partial_{t} U_{K}(t)=U_{K}(t) \tau_{\mathcal{R}}^{-t}(K), \quad U_{K}(0)=\mathbb{1} .
$$

Hence, we have

$$
\omega_{\mathrm{i}} \circ \tau_{K}^{t}=\omega_{\mathrm{i}} \circ \alpha_{U_{K}(t)},
$$

and we can apply the results of the previous section. Assuming $K \in \operatorname{Dom}\left(\delta_{\mathcal{R}}\right)$, it follows from the Dyson expansion

$$
U_{K}(t)=\mathbb{1}+\sum_{n=1}^{\infty}(-\mathrm{i})^{n} \int_{0 \leq s_{1} \leq \cdots \leq s_{n} \leq t} \tau_{\mathcal{R}}^{-s_{1}}(K) \cdots \tau_{\mathcal{R}}^{-s_{n}}(K) \mathrm{d} s_{1} \cdots \mathrm{d} s_{n}
$$

that $U_{K}(t) \in \operatorname{Dom}\left(\delta_{\mathcal{R}}\right)$ for all $t \in \mathbb{R}$ and Eq. (3.12) gives

$$
\Delta S(K, t)+\sigma(K, t)=\beta \Delta \mathcal{Q}(K, t),
$$

where

$$
\Delta \mathcal{Q}(K, t)=-\mathrm{i} \omega_{\mathrm{i}}\left(U_{K}^{*}(t) \delta_{\mathcal{R}}\left(U_{K}(t)\right)\right)
$$

and

$$
\Delta S(K, t)=S\left(\rho_{\mathrm{i}}\right)-S\left(\rho_{K}(t)\right), \quad \sigma(K, t)=S\left(\omega_{\mathrm{i}} \circ \tau_{K}^{t} \mid \rho_{K}(t) \otimes \nu_{\mathrm{i}}\right),
$$

$\rho_{K}(t)$ denoting the restriction of $\omega_{\mathrm{i}} \circ \tau_{K}^{t}$ to $\mathcal{O}_{\mathcal{S}}$.

Remark. One easily checks that $T(t)=\mathrm{i} U_{K}^{*}(t) \delta_{\mathcal{R}}\left(U_{K}(t)\right)+\tau_{\mathcal{R}}^{-t}(K)$ satisfies the Cauchy problem

$$
\partial_{t} T(t)=\mathrm{i}\left[\tau_{\mathcal{R}}^{-t}(K), T(t)\right], \quad T(0)=K .
$$

Comparing with Eq. (4.21), we infer $T(t)=U_{K}^{*}(t) K U_{K}(t)$, so that

$$
-\mathrm{i} U_{K}^{*}(t) \delta_{\mathcal{R}}\left(U_{K}(t)\right)=\tau_{\mathcal{R}}^{-t}\left(K-\tau_{K}^{t}(K)\right),
$$


and therefore

$$
\Delta \mathcal{Q}(K, t)=\omega_{\mathrm{i}}\left(K-\tau_{K}^{t}(K)\right) .
$$

Conservation of the total energy leads to the conclusion that $\Delta \mathcal{Q}(K, t)$ is indeed the change of the reservoir energy. Since

$$
\partial_{t}\left(K-\tau_{K}^{t}(K)\right)=\tau_{K}^{t}\left(-\delta_{\mathcal{R}}(K)\right)
$$

one can further write

$$
\Delta \mathcal{Q}(K, t)=-\int_{0}^{t} \omega_{\mathrm{i}}\left(\tau_{K}^{s}(\Phi)\right) \mathrm{d} s
$$

where

$$
\Phi=\delta_{\mathcal{R}}(K),
$$

is the observable describing the instantaneous energy flux out of $\mathcal{R}$.

\subsection{The Landauer principle in the large time limit}

We shall now consider realizations of the state transition $\rho_{\mathrm{i}} \rightarrow \rho_{\mathrm{f}}$ and the corresponding entropic balance as a limiting case of the transition $\rho_{\mathrm{i}} \rightarrow \rho_{K}(t)$ as $t \rightarrow \infty$. To simplify the discussion, we shall assume here and in the following that the equilibrium state $\nu_{\mathrm{i}}$ describes a pure thermodynamic phase of $\mathcal{R}$, i.e., that it is an extremal $\left(\tau_{\mathcal{R}}, \beta\right)$-KMS state. This implies that for $K \in \mathcal{O}^{\text {sa }}$ there is a unique $\left(\tau_{K}, \beta\right)$-KMS state in $\mathcal{N}_{\omega_{\mathrm{i}}}$ which we denote by $\mu_{K}$. Let $\varrho_{K}$ be its restriction to $\mathcal{O}_{\mathcal{S}}$. We observe that $\mathcal{N}_{\mu_{K}}=\mathcal{N}_{\omega_{\mathrm{i}}}$.

The following proposition shows that by an appropriate choice of $K$ we can reach any faithful ${ }^{5}$ target state of $\mathcal{O}_{\mathcal{S}}$ with the $\left(\tau_{K}, \beta\right)$-KMS state $\mu_{K}$.

Proposition 4.1 Let $\rho_{\mathrm{f}}>0$ be a state on $\mathcal{O}_{\mathcal{S}}$ and $V \in \mathcal{O}^{\mathrm{sa}}$. Then there exists $\delta>0$ and a real analytic function $]-\delta, \delta\left[\ni \lambda \mapsto H_{\lambda} \in \mathcal{O}_{\mathcal{S}}^{\text {sa }}\right.$ such that $H_{0}=-\beta^{-1} \log \rho_{\mathrm{f}}$ and $\varrho_{K_{\lambda}}=\rho_{\mathrm{f}}$ for $K_{\lambda}=H_{\lambda}+\lambda V$ and any $\lambda \in]-\delta, \delta[$.

Our main dynamical assumption is:

Assumption A. There exists $\gamma \in]-\delta, \delta\left[\right.$ such that the KMS state $\mu_{K_{\gamma}}$ is mixing for the dynamical system $\left(\mathcal{O}, \tau_{K_{\gamma}}\right)$.

We now explore the consequences of this assumption on the long time asymptotics of entropy balance (note that obviously $\gamma \neq 0$ ). The first is

$$
\lim _{t \rightarrow \infty} \rho_{K_{\gamma}}(t)=\rho_{\mathrm{f}}
$$

Furthermore,

$$
\begin{gathered}
\Delta S=\lim _{t \rightarrow \infty} \Delta S\left(K_{\gamma}, t\right)=S\left(\rho_{\mathrm{i}}\right)-S\left(\rho_{\mathrm{f}}\right), \\
\Delta \mathcal{Q}(\gamma)=\lim _{t \rightarrow \infty} \Delta \mathcal{Q}\left(K_{\gamma}, t\right)=\omega_{\mathrm{i}}\left(K_{\gamma}\right)-\mu_{K_{\gamma}}\left(K_{\gamma}\right) .
\end{gathered}
$$

It follows from (4.22) that

$$
\sigma(\gamma)=\lim _{t \rightarrow \infty} \sigma\left(K_{\gamma}, t\right)
$$

also exists and that

$$
\Delta S+\sigma(\gamma)=\beta \Delta \mathcal{Q}(\gamma)
$$

Clearly, $\sigma(\gamma) \geq 0$, and the relation (4.24) gives the Landauer principle for the transition process $\rho_{\mathrm{i}} \rightarrow \rho_{\mathrm{f}}$ realized by the large time limit $t \rightarrow \infty$.

One does not expect that the Landauer bound can be saturated by an instantaneously switched interaction and that is indeed the case.

\footnotetext{
${ }^{5}$ The cases where the target state is not faithful are handled by an additional limiting argument that we will describe later.
} 


\section{Proposition 4.2}

$$
\sigma(\gamma)>0
$$

This completes our analysis of the Landauer principle for instantaneously switched interactions.

Remark 1. The above analysis extends with no changes to $W^{*}$-dynamical systems. Unbounded interactions $V$ satisfying the general assumptions of [DJP] are also allowed.

Remark 2. In the Landauer erasing principle, $\rho_{\mathrm{i}}=\mathbb{1} / d$ and $\rho_{\mathrm{f}}=|\psi\rangle\langle\psi|$. Pure target states are thermodynamically singular and cannot be directly reached by the action of a thermal reservoir unless the reservoir is at zero temperature. The proper way to formulate the Landauer principle for pure states is to examine the stability of the entropy balance equation of the processes with faithful target states $\rho_{\mathrm{f}}^{\prime}$ in a vicinity of $\rho_{\mathrm{f}}$. For instantaneously switched interactions there is no stability. As $\rho_{\mathrm{f}}^{\prime} \rightarrow \rho_{\mathrm{f}}, S\left(\rho_{\mathrm{f}}^{\prime}\right) \rightarrow S\left(\rho_{\mathrm{f}}\right)=0$. However, in this limit $\sigma(\gamma) \rightarrow \infty$ and $\Delta \mathcal{Q}(\gamma) \rightarrow \infty$. This singularity is due to an instantaneous change of the Hamiltonian. As we shall see in the next section, if the change of the Hamiltonian is adiabatic, this singularity is absent.

Remark 3. It follows from Araki's perturbation theory of KMS states that the map

$$
]-\delta, \delta\left[\ni \lambda \mapsto \Delta \mathcal{Q}(\lambda)=\omega_{\mathrm{i}}\left(K_{\lambda}\right)-\mu_{K_{\lambda}}\left(K_{\lambda}\right)\right.
$$

is real analytic and that

$$
\Delta \mathcal{Q}(0)=\rho_{\mathrm{i}}\left(H_{0}\right)-\rho_{\mathrm{f}}\left(H_{0}\right) .
$$

The relation (4.24) defines $\sigma(\lambda)$ for $\lambda \in]-\delta, \delta[$ and

$$
\sigma(0)=S\left(\rho_{\mathrm{i}} \mid \rho_{\mathrm{f}}\right)
$$

Remark 4. For many models, Assumption $\mathbf{A}$ is satisfied in a stronger form:

Assumption A'. There exists $\lambda_{0}>0$ such that for $0<|\lambda|<\lambda_{0}$ the KMS state $\mu_{K_{\lambda}}$ is mixing for the dynamical system $\left(\mathcal{O}, \tau_{K_{\lambda}}\right)$.

In this case the entropy balance equation

$$
\Delta S+\sigma(0)=\beta \Delta \mathcal{Q}(0)
$$

gives the Landauer principle for the transition process $\rho_{\mathrm{i}} \rightarrow \rho_{\mathrm{f}}$ realized by the double limit $t \rightarrow \infty$, $\lambda \rightarrow 0$. The relation (4.26) is certainly expected in view of the Lebowitz-Spohn weak coupling limit thermodynamics of open quantum systems [LeSp, JPW]. Under suitable assumptions, in the van Hove scaling limit $\lambda \rightarrow 0, t \rightarrow \infty$ with $\bar{t}=\lambda^{2} t$ fixed, the reduced dynamics of $\mathcal{S}$ is described by a quantum dynamical semigroup on $\mathcal{O}_{\mathcal{S}}$,

$$
T_{\bar{t}}(A)=\mathrm{e}^{\bar{t} \mathcal{K}}(A),
$$

where $\mathcal{K}$ is the so-called Davies generator in the Heisenberg picture. Under the usual effective coupling assumptions one has ${ }^{6}$

$$
\lim _{\bar{t} \rightarrow \infty} \mathrm{e}^{\bar{t} \mathcal{K}^{\dagger}}(\rho)=\rho_{\mathrm{f}}
$$

for any state $\rho$ on $\mathcal{O}_{\mathcal{S}}$. This relation defines the transition process $\rho_{\mathrm{i}} \rightarrow \rho_{\mathrm{f}}$ in the van Hove scaling limit. The Lebowitz-Spohn entropy balance equation is

$$
S\left(\rho_{\mathrm{i}}\right)-S\left(\mathrm{e}^{\bar{t} \mathcal{K}^{\dagger}}\left(\rho_{\mathrm{i}}\right)\right)+S\left(\rho_{\mathrm{i}} \mid \mathrm{e}^{\bar{t} \mathcal{K}^{\dagger}}\left(\rho_{\mathrm{i}}\right)\right)=\beta \Delta \overline{\mathcal{Q}}(\bar{t}),
$$

\footnotetext{
${ }^{6}$ The adjoint is taken with respect to the inner product $\langle A, B\rangle=\operatorname{tr}\left(A^{*} B\right)$ on $\mathcal{O}_{\mathcal{S}}$
} 
where

$$
\Delta \overline{\mathcal{Q}}(\bar{t})=\rho_{\mathrm{i}}\left(\mathrm{e}^{\bar{t} \mathcal{K}}\left(H_{0}\right)-H_{0}\right)
$$

It follows that

$$
\Delta \overline{\mathcal{Q}}=\lim _{\bar{t} \rightarrow \infty} \Delta \overline{\mathcal{Q}}(\bar{t})=\rho_{\mathrm{f}}\left(H_{0}\right)-\rho_{\mathrm{i}}\left(H_{0}\right),
$$

and one derives

$$
\beta \Delta \overline{\mathcal{Q}}=S\left(\rho_{\mathrm{i}} \mid \rho_{\mathrm{f}}\right)+S\left(\rho_{\mathrm{i}}\right)-S\left(\rho_{\mathrm{f}}\right) .
$$

Since the van Hove weak coupling limit is expected to yield the first non-trivial contribution (in the coupling constant $\lambda$ ) to the microscopic thermodynamics, the identity $(4.26)=(4.27)$ is certainly not surprising. A somewhat surprising fact is that Assumption $\mathbf{A}^{\prime}$ is only vaguely related to the assumptions of the weak coupling limit theory [Dav, DF, LeSp].

Remark 5. Specific physically relevant models (spin-boson model, spin-fermion model, electronic black box model, locally interacting fermionic systems) for which Assumption $\mathbf{A}^{\prime}$ holds are discussed in [AM, AJPP1, AJPP2, BFS, BM, dRK, DJ, FMU, FMSU, JOP1, JOP2, JP1, JP6, MMS1, MMS2].

\section{Adiabatically switched interactions}

Our next topic is the optimality of the Landauer bound in the context of time dependent Hamiltonian dynamics of $\mathcal{S}+\mathcal{R}$. We shall assume that the reader is familiar with basic results concerning non-autonomous perturbations of $C^{*}$-dynamical systems (see Section 5.4.4 in [BR2] and the Appendix to Section IV.5 in $[\mathrm{Si}])$.

\subsection{Setup}

Let $K:[0,1] \rightarrow \mathcal{O}^{\text {sa }} \cap \operatorname{Dom}\left(\delta_{\mathcal{R}}\right)$ be a continuous function which we assume to be twice continuously differentiable on $] 0,1[$ with uniformly bounded first and second derivatives. For $T>0$, we define the rescaled function $K_{T}$ by

$$
K_{T}(t)=K(t / T)
$$

Let $[0, T] \ni t \mapsto \alpha_{K_{T}}^{t}$ be the non-autonomous $C^{*}$-dynamics defined by the Cauchy problem

$$
\partial_{t} \alpha_{K_{T}}^{t}(A)=\alpha_{K_{T}}^{t}\left(\delta_{\mathcal{R}}(A)+\mathrm{i}\left[K_{T}(t), A\right]\right), \quad \alpha_{K_{T}}^{0}(A)=A .
$$

We recall that $\left\{\alpha_{K_{T}}^{t}\right\}_{t \in[0, T]}$ is a strongly continuous family of $*$-automorphisms of $\mathcal{O}$ given by

$$
\alpha_{K_{T}}^{t}(A)=\tau_{\mathcal{R}}^{t}(A)+\sum_{n=1}^{\infty} \int_{0 \leq s_{1} \leq \cdots \leq s_{n} \leq t} \mathrm{i}\left[\tau_{\mathcal{R}}^{s_{1}}\left(K_{T}\left(s_{1}\right)\right), \mathrm{i}\left[\cdots, \mathrm{i}\left[\tau_{\mathcal{R}}^{s_{n}}\left(K_{T}\left(s_{n}\right)\right), \tau_{\mathcal{R}}^{t}(A)\right]\right]\right] \mathrm{d} s_{1} \cdots \mathrm{d} s_{n} .
$$

Moreover, the interaction representation

$$
\tau_{\mathcal{R}}^{-t} \circ \alpha_{K_{T}}^{t}(A)=\tau_{\mathcal{R}}^{-t}\left(\Gamma_{K_{T}}(t)\right)^{*} A \tau_{\mathcal{R}}^{-t}\left(\Gamma_{K_{T}}(t)\right),
$$

holds with a family of unitaries $\Gamma_{K_{T}}(t) \in \mathcal{O}$ satisfying the Cauchy problem

$$
\mathrm{i} \partial_{t} \Gamma_{K_{T}}(t)=\tau_{\mathcal{R}}^{t}\left(K_{T}(t)\right) \Gamma_{K_{T}}(t), \quad \Gamma_{K_{T}}(0)=\mathbb{1} .
$$

We denote by $\rho_{T}$ the restriction of $\omega_{\mathrm{i}} \circ \alpha_{K_{T}}^{T}$ to $\mathcal{O}_{\mathcal{S}}$. Our assumptions ensure that $\Gamma_{K_{T}}(T) \in \operatorname{Dom}\left(\delta_{\mathcal{R}}\right)$ and Eq. (3.12) gives

$$
\Delta S_{T}+\sigma_{T}=\beta \Delta \mathcal{Q}_{T},
$$

where

$$
\Delta S_{T}=S\left(\rho_{\mathrm{i}}\right)-S\left(\rho_{T}\right), \quad \Delta \mathcal{Q}_{T}=-\mathrm{i} \omega_{\mathrm{i}}\left(\Gamma_{K_{T}}^{*}(T) \delta_{\mathcal{R}}\left(\Gamma_{K_{T}}(T)\right)\right)
$$


and

$$
\sigma_{T}=S\left(\omega_{\mathrm{i}} \circ \alpha_{K_{T}}^{T} \mid \rho_{T} \otimes \nu_{\mathrm{i}}\right) .
$$

To interpret the right hand side of Eq. (5.31), we write

$$
\Delta \mathcal{Q}_{T}=Q(T)-Q(0)=\int_{0}^{T} \partial_{t} Q(t) \mathrm{d} t
$$

with $Q(t)=-\mathrm{i} \omega_{\mathrm{i}}\left(\Gamma_{K_{T}}^{*}(t) \delta_{\mathcal{R}}\left(\Gamma_{K_{T}}(t)\right)\right)$. It follows from the differential equation (5.30) that

$$
\partial_{t} Q(t)=-\omega_{\mathrm{i}}\left(\Gamma_{K_{T}}^{*}(t) \tau_{\mathcal{R}}^{t}\left(\delta_{\mathcal{R}}\left(K_{T}(t)\right)\right) \Gamma_{K_{T}}(t)\right),
$$

and Eq. (5.28)-(5.29) give

$$
\begin{aligned}
\partial_{t} Q(t) & =-\omega_{\mathrm{i}} \circ \alpha_{K_{T}}^{t}\left(\delta_{\mathcal{R}}\left(K_{T}(t)\right)\right) \\
& =-\frac{\mathrm{d}}{\mathrm{d} t} \omega_{\mathrm{i}} \circ \alpha_{K_{T}}^{t}\left(K_{T}(t)\right)+\omega_{\mathrm{i}} \circ \alpha_{K_{T}}^{t}\left(\frac{\mathrm{d}}{\mathrm{d} t} K_{T}(t)\right) .
\end{aligned}
$$

This leads to

$$
\Delta \mathcal{Q}_{T}+\omega_{\mathrm{i}} \circ \alpha_{K_{T}}^{T}\left(K_{T}(T)\right)-\omega_{\mathrm{i}}\left(K_{T}(0)\right)=\int_{0}^{T} \omega_{\mathrm{i}} \circ \alpha_{K_{T}}^{t}\left(P_{T}(t)\right) \mathrm{d} t,
$$

where $P_{T}(t)=\partial_{t} K_{T}(t)$ is the instantaneous power injected into the system $\mathcal{S}+\mathcal{R}$. Energy conservation yields that $\Delta \mathcal{Q}_{T}$ is the total change in the energy of the subsystem $\mathcal{R}$ from time $t=0$ to time $t=T$.

\subsection{The Landauer principle in the adiabatic limit}

We shall now consider the adiabatic limit $T \rightarrow \infty$. Our main assumption in this section concerns the instantaneous $C^{*}$-dynamics $\tau_{K(\gamma)}$.

Assumption B. For $0<\gamma<1$, the $\left(\tau_{K(\gamma)}, \beta\right)$-KMS state $\mu_{K(\gamma)}$ is ergodic for the dynamical $\operatorname{system}\left(\mathcal{O}, \tau_{K(\gamma)}\right)$.

The Avron-Elgart adiabatic theorem [AE, Teu] and Araki's perturbation theory of KMS states give ([ASF1][ASF3], [JP8]):

Theorem 5.1 Suppose that Assumption $\mathbf{B}$ holds. Then one has

$$
\lim _{T \rightarrow \infty}\left\|\mu_{K(0)} \circ \alpha_{K_{T}}^{\gamma T}-\mu_{K(\gamma)}\right\|=0
$$

for all $\gamma \in[0,1]$.

For completeness and the reader's convenience the proof of Theorem 5.1 is given in Section 7.

Let $\rho_{\mathrm{f}}$ be a given faithful target state of $\mathcal{S}$ and set

$$
\omega_{\mathrm{f}}=\rho_{\mathrm{f}} \otimes \nu_{\mathrm{i}}
$$

According to Theorem 5.1, to achieve the transition $\rho_{\mathrm{i}} \rightarrow \rho_{\mathrm{f}}$ in the limit $T \rightarrow \infty$, it suffices to assume that Assumption $\mathbf{B}$ holds for $K(\gamma)$ satisfying the boundary conditions

$$
K(0)=-\beta^{-1} \log \rho_{\mathrm{i}}, \quad K(1)=-\beta^{-1} \log \rho_{\mathrm{f}} .
$$


Indeed, these conditions ensure that $\mu_{K(0)}=\omega_{\mathrm{i}}$ and $\mu_{K(1)}=\omega_{\mathrm{f}}$ so that

$$
\lim _{T \rightarrow \infty} \omega_{\mathrm{i}} \circ \alpha_{K_{T}}^{T}=\lim _{T \rightarrow \infty} \mu_{K(0)} \circ \alpha_{K_{T}}^{T}=\mu_{K(1)}=\omega_{\mathrm{f}} .
$$

Theorem 5.1 further implies that

$$
\Delta S=\lim _{T \rightarrow \infty} \Delta S_{T}=S\left(\rho_{\mathrm{i}}\right)-S\left(\rho_{\mathrm{f}}\right) .
$$

Moreover, rewriting Eq. (5.32) as

$$
\Delta \mathcal{Q}_{T}=\int_{0}^{1} \omega_{\mathrm{i}} \circ \alpha_{K_{T}}^{\gamma T}\left(\partial_{\gamma} K(\gamma)\right) \mathrm{d} \gamma-\beta^{-1} \omega_{\mathrm{i}} \circ \alpha_{K_{T}}^{T}\left(\log \rho_{\mathrm{f}}\right)+\beta^{-1} \omega_{\mathrm{i}}\left(\log \rho_{\mathrm{i}}\right),
$$

we get

$$
\Delta \mathcal{Q}=\lim _{T \rightarrow \infty} \Delta \mathcal{Q}_{T}=\int_{0}^{1} \mu_{K(\gamma)}\left(\partial_{\gamma} K(\gamma)\right) \mathrm{d} \gamma+\beta^{-1} \Delta S .
$$

The balance equation (5.31) yields that

$$
\Delta S+\sigma=\beta \Delta \mathcal{Q}
$$

with

$$
\sigma=\lim _{T \rightarrow \infty} \sigma_{T}=\beta \int_{0}^{1} \mu_{K(\gamma)}\left(\partial_{\gamma} K(\gamma)\right) \mathrm{d} \gamma
$$

Clearly, $\sigma \geq 0$. The adiabatic limit is a quasi-static process and one may expect the optimality of the Landauer bound. This is indeed the case.

\section{Proposition 5.2}

$$
\sigma=0
$$

The proof of the last result requires modular theory. Note however that for finite reservoirs the relation

$$
\int_{0}^{1} \mu_{K(\gamma)}\left(\partial_{\gamma} K(\gamma)\right) \mathrm{d} \gamma=0
$$

is easily derived ${ }^{7}$ :

$$
\begin{aligned}
\int_{0}^{1} \mu_{K(\gamma)}\left(\partial_{\gamma} K(\gamma)\right) \mathrm{d} \gamma & =\int_{0}^{1} \frac{\operatorname{tr}\left(\mathrm{e}^{-\beta\left(H_{\mathcal{R}}+K(\gamma)\right)} \partial_{\gamma} K(\gamma)\right)}{\operatorname{tr}\left(\mathrm{e}^{-\beta\left(H_{\mathcal{R}}+K(\gamma)\right)} \mathrm{d} \gamma\right.} \\
& =-\frac{1}{\beta} \int_{0}^{1} \partial_{\gamma} \log \operatorname{tr}\left(\mathrm{e}^{-\beta\left(H_{\mathcal{R}}+K(\gamma)\right)}\right) \mathrm{d} \gamma \\
& =-\frac{1}{\beta}\left(\log \operatorname{tr}\left(\omega_{\mathrm{f}}\right)-\log \operatorname{tr}\left(\omega_{\mathrm{i}}\right)\right)=0 .
\end{aligned}
$$

This completes our mathematical analysis of the Landauer principle for adiabatically switched interactions.

Remark 1. Regarding the remarks at the end of Section 4.2, Remark 1 applies to the results of this section as well. In the adiabatic case the entropy production term vanishes and the instability discussed in Remark 2 is absent. Remark 4 also extends to the adiabatic setting (see [DS] for the discussion of the adiabatic theorem and $[\mathrm{AHHH}$ ] for a discussion of the Landauer principle in the van Hove weak coupling limit). Since mixing implies ergodicity, the physically relevant models for which Assumption $\mathbf{B}$ has been verified are listed in Remark 5.

Remark 2. The Narnhoffer-Thirring adiabatic theorem of quantum statistical mechanics [NT] is based on $C^{*}$-scattering and requires $L^{1}$-asymptototic Abelianess which is stronger than our ergodicity assumption B. The physically relevant models satisfying $L^{1}$-asymptotic Abelianess are discussed in [AM, AJPP1, AJPP2, BM, FMU, FMSU, JOP2]. If $L^{1}$-asymptotic Abelianess holds, then the Landauer principle for adiabatically switched interactions can be further refined. The result of this analysis is given in [Han].

\footnotetext{
${ }^{7}$ On the other hand, Theorem 5.1 and relation $\lim _{T \rightarrow \infty} \sigma_{T}=\sigma$ cannot hold for finite reservoirs.
} 


\section{Discussion}

In this section we comment on the key ingredients involved in the analysis of the Landauer principle presented in Sections 3-5, and on their relation with the work [ReWo].

The entropy balance equation. Relation (3.9) is a model-independent structural identity linked to the KMS condition and modular theory. It is tautological in the finite dimensional case. The general case follows from Araki's perturbation theory of the modular structure. The mathematical analysis of the second law of thermodynamics starts with the entropy balance equation but certainly does not end there ${ }^{8}$ ASF1, ASF2, ASF3]. The thermodynamic behavior of the coupled system $\mathcal{S}+\mathcal{R}$ emerges only in the thermodynamic limit in which the reservoir $\mathcal{R}$ becomes infinitely extended. In the large time limit the coupled system settles into a steady state, substantiating the zeroth law of thermodynamics [BFS, DJ, FM, JP1].

These two limiting processes, large reservoir size and large time, have been pillars of the mathematical theory of open quantum systems since its foundations [Rob, BR1, BR2]. In a sense, the same applies to the Landauer principle and this is the main message of this note: the control of the entropy balance equation for open quantum systems with infinitely extended reservoirs in the large time (or adiabatic) limit is one of the central issues in the analysis of the Landauer principle within quantum statistical mechanics. This brings us to our second point.

Confined reservoirs. A typical physical example of a confined reservoir is a Fermi gas or a Bose gas in thermal equilibrium confined to a finite box. Confined reservoirs are not ergodic and lead to quasi-periodic dynamics when coupled to a finite system $\mathcal{S}$. The analysis of the large time asymptotics of such systems requires some time averaging which is not compatible with the formulation of Landauer's principle. In this context, one may say that the main contribution of [ReWo] concerns estimates regarding the accuracy of the Landauer principle for confined reservoirs.

Ergodicity. The large time asymptotics of the microscopic system $\mathcal{S}$ coupled to the thermal reservoir $\mathcal{R}$ is critically linked to the ergodic properties (Assumptions $\mathbf{A}$ and $\mathbf{B}$ ) of the dynamical system which describes the joint system $\mathcal{S}+\mathcal{R}$ in the framework of statistical mechanics. As we have shown, ergodicity allows for arbitrary transition $\rho_{\mathrm{i}} \rightarrow \rho_{\mathrm{f}}$ of the system $\mathcal{S}$ in the adiabatic limit with the minimal energy dissipation predicted by Landauer. Needless to say, Assumptions A and $\mathbf{B}$, which are part of the zeroth law of thermodynamics, are notoriously difficult to prove for physically relevant models. In particular, they cannot hold in the framework of [ReWo], where the reservoirs are confined.

Conclusion. The claim of the authors in [ReWo] that they have proven the Landauer principle in quantum statistical mechanics may lead to a confusion regarding some foundational aspects of mathematical theory of open quantum systems and we have attempted to clarify this point. The complementary analysis of the Landauer principle presented in Sections 3-5 relies on the entropy balance equation, Araki's perturbation theory of KMS states, and the Avron-Elgart adiabatic theorem. It is a simple consequence of well-known and deep structural results. The workers in quantum information theory appear unaware of this fact. From the point of view of state-of-the-art quantum statistical mechanics, the interesting aspect of the Landauer principle concerns the verifications of Assumptions A and $\mathbf{B}$. The models for which this has been achieved are discussed in [AM, AJPP1, AJPP2, BFS, BM, dRK, DJ, FMU, FMSU, JOP1, JOP2, JP1, JP6, MMS1, MMS2]. One may say that one of the main challenges of quantum statistical mechanics at the moment is to extend the class of physically relevant models for which Assumptions A and $\mathbf{B}$ can be proved. The progress in this direction requires novel ideas and techniques in the study of the large time dynamics of infinitely extended Hamiltonian quantum statistical models.

\footnotetext{
${ }^{8}$ In the literature, the entropy balance equation (3.9) is sometimes called "finite time second law of thermodynamics" reflecting the fact that in typical application $U$ is a unitary cocycle describing time evolution over a finite time period.
} 


\section{Proofs}

Preliminaries. We start with some general properties of the GNS representation $(\mathfrak{H}, \pi, \Omega)$ of $\mathcal{O}$ associated to the state $\omega_{\mathrm{i}}=\rho_{\mathrm{i}} \otimes \nu_{\mathrm{i}}$. This material is standard and we refer the reader to [BR1, BR2, DJP] for a detailed exposition and proofs. We denote by $\mathfrak{M}=\pi(\mathcal{O})^{\prime \prime}$ the enveloping von Neumann algebra and by $\mathcal{P} \subset \mathfrak{H}$ and $J$ the natural cone and modular conjugation of the pair $(\mathfrak{M}, \Omega)$. Any state $\omega \in \mathcal{N}_{\omega_{\mathrm{i}}}$ has a unique standard representative, a unit vector $\Psi \in \mathcal{P}$ such that $\omega(A)=\langle\Psi, \pi(A) \Psi\rangle$ for all $A \in \mathcal{O}$. The standard Liouvillean of a strongly continuous group $\varsigma$ of $*$-automorphisms of $\mathcal{O}$ is the unique self-adjoint operator $L$ on $\mathfrak{H}$ such that

$$
\pi\left(\varsigma^{t}(A)\right)=\mathrm{e}^{\mathrm{i} t L} \pi(A) \mathrm{e}^{-\mathrm{i} t L}, \quad \mathrm{e}^{\mathrm{i} t L} \mathcal{P} \subset \mathcal{P},
$$

for all $t \in \mathbb{R}$ and all $A \in \mathcal{O}$.

Let $L_{0}$ be the standard Liouvillean of a group $\varsigma_{0}^{t}=\mathrm{e}^{t \delta_{0}}$ of $*$-automorphisms of $\mathcal{O}$ and $\Phi_{0}$ the standard representative of a $\left(\varsigma_{0}, \beta\right)$-KMS state $\omega_{0} \in \mathcal{N}_{\omega_{\mathrm{i}}}$. If $Q \in \mathcal{O}^{\text {sa }}$ and $\delta_{Q}=\delta_{0}+\mathrm{i}[Q, \cdot]$, then the standard Liouvillean of the locally perturbed group $\varsigma_{Q}^{t}=\mathrm{e}^{t \delta_{Q}}$ is

$$
L_{Q}=L_{0}+\pi(Q)-J \pi(Q) J
$$

Moreover, $\Phi_{0} \in \operatorname{Dom}\left(\mathrm{e}^{-\beta\left(L_{0}+\pi(Q)\right) / 2}\right)$ and the vector

$$
\Psi_{Q}=\frac{\Phi_{Q}}{\left\|\Phi_{Q}\right\|}, \quad \Phi_{Q}=\mathrm{e}^{-\beta\left(L_{0}+\pi(Q)\right) / 2} \Phi_{0},
$$

is the standard representative of a $\left(\varsigma_{Q}, \beta\right)$-KMS state. In particular, one has $\Psi_{Q} \in \operatorname{Ker}\left(L_{Q}\right)$.

We shall need the following perturbative expansion of the unnormalized KMS vector $\Phi_{Q}$. For any $Q_{1}, \ldots$, $Q_{n} \in \mathfrak{M}$ and $\left(\beta_{1}, \ldots, \beta_{n}\right) \in T_{\beta, n}=\left\{\left(\beta_{1}, \ldots, \beta_{n}\right) \in \mathbb{R}_{+}^{n}, \mid \beta_{1}+\cdots+\beta_{n} \leq \beta / 2\right\}$ one has

$$
\Phi_{0} \in \operatorname{Dom}\left(\mathrm{e}^{-\beta_{1} L_{0}} Q_{1} \cdots \mathrm{e}^{-\beta_{n} L_{0}} Q_{n}\right) .
$$

Moreover, the map

$$
T_{\beta, n} \ni\left(\beta_{1}, \ldots, \beta_{n}\right) \mapsto \mathrm{e}^{-\beta_{1} L_{0}} Q_{1} \cdots \mathrm{e}^{-\beta_{n} L_{0}} Q_{n} \Phi_{0} \in \mathfrak{H},
$$

is continuous and satisfies

$$
\sup _{\left(\beta_{1}, \ldots, \beta_{n}\right) \in T_{\beta, n}}\left\|\mathrm{e}^{-\beta_{1} L_{0}} Q_{1} \cdots \mathrm{e}^{-\beta_{n} L_{0}} Q_{n} \Phi_{0}\right\| \leq\left\|Q_{1}\right\| \cdots\left\|Q_{n}\right\| .
$$

The vector $\Phi_{Q}$ has the norm convergent expansion

$$
\Phi_{Q}=\sum_{n=0}^{\infty}(-1)^{n} \int_{T_{\beta, n}} \mathrm{e}^{-\beta_{1} L_{0}} \pi(Q) \cdots \mathrm{e}^{-\beta_{n} L_{0}} \pi(Q) \Phi_{0} \mathrm{~d} \beta_{1} \cdots \mathrm{d} \beta_{n} .
$$

For $\delta Q \in \mathcal{O}^{\text {sa }}$, the following chain rule applies

$$
\Phi_{Q+\delta Q}=\mathrm{e}^{-\beta\left(L_{Q}+\pi(\delta Q)\right) / 2} \Phi_{Q},
$$

(see Theorem 5.1 (6) in [DJP]). It follows from the expansion (7.34) and the estimate (7.33) that the map $\mathcal{O}^{\text {sa }} \ni Q \mapsto \Phi_{Q} \in \mathfrak{H}$ is differentiable. Its derivative at $Q$ is the map

$$
\Phi_{Q}^{\prime}: \delta Q \mapsto-\int_{0}^{\beta / 2} \mathrm{e}^{-s L_{Q}} \pi(\delta Q) \Phi_{Q} \mathrm{~d} s
$$

The same argument shows that if $\alpha \mapsto Q(\alpha)$ is a real analytic function from some open subset of $\mathbb{R}^{n}$ to $\mathcal{O}^{\text {sa }}$, then the function $\alpha \mapsto \Phi_{Q(\alpha)}$ is also real analytic. 
Proof of Proposition 3.2 To simplify the notation, we write $\rho=\rho_{\mathrm{i}}, \nu=\nu_{\mathrm{i}}, \omega=\omega_{\mathrm{i}}$. We denote by $\Omega_{U} \in \mathcal{P}$ the standard representative of the state $\omega_{U}$. The modular operator $\Delta_{\omega}$ and the relative modular operator $\Delta_{\omega_{U} \mid \omega}$ are positive operators on $\mathfrak{H}$ satisfying

$$
J \Delta_{\omega}^{1 / 2} \pi(A) \Omega=\pi(A)^{*} \Omega, \quad J \Delta_{\omega_{U} \mid \omega}^{1 / 2} \pi(A) \Omega=\pi(A)^{*} \Omega_{U}
$$

for all $A \in \mathcal{O}$. It follows from $\omega_{U}=\omega \circ \alpha_{U}$ that $\Omega_{U}=\pi(U) J \pi(U) J \Omega$. Since $J \pi(U) J \in \mathfrak{M}^{\prime}$, one has

$$
\begin{aligned}
J \Delta_{\omega_{U} \mid \omega}^{1 / 2} \pi(A) \Omega & =\pi(A)^{*} \pi(U) J \pi(U) J \Omega \\
& =J \pi(U) J \pi(A)^{*} \pi(U) \Omega \\
& =J \pi(U) J J \Delta_{\omega}^{1 / 2} \pi(U)^{*} \pi(A) \Omega
\end{aligned}
$$

and the cyclic property of $\Omega$ allows us to conclude that

$$
\Delta_{\omega_{U} \mid \omega}=\pi(U) \Delta_{\omega} \pi(U)^{*}
$$

The product structure of the state $\omega$ induces the factorization $\mathfrak{H}=\mathfrak{H}_{\mathcal{S}} \otimes \mathfrak{H}_{\mathcal{R}}$ where $\mathfrak{H}_{\mathcal{S}}=\mathcal{O}_{\mathcal{S}}$ equipped with the inner product $\langle X, Y\rangle=\operatorname{tr}\left(X^{*} Y\right)$ and the Hilbert space $\mathfrak{H}_{\mathcal{R}}$ carries a GNS representation of $\mathcal{O}_{\mathcal{R}}$ induced by the state $\nu$. Moreover, one has

$$
\Delta_{\omega}=\Delta_{\rho} \otimes \Delta_{\nu}, \quad \Delta_{\omega_{U} \mid \omega}=\Delta_{\rho_{U} \mid \rho} \otimes \Delta_{\nu},
$$

where $\Delta_{\rho}, \Delta_{\nu}$ and $\Delta_{\rho_{U} \mid \rho}$ are respectively the modular operator of the state $\rho$, the modular operator of the state $\nu$, and the relative modular operator of the state $\rho_{U}$ w.r.t. $\rho$. The operators $\Delta_{\rho}$ and $\Delta_{\rho_{U}} \mid \rho$ act on $\mathfrak{H}_{\mathcal{S}}$ according to

$$
\Delta_{\rho} X=\rho X \rho^{-1}, \quad \Delta_{\rho_{U} \mid \rho} X=\rho_{U} X \rho^{-1}
$$

(see, e.g., Section 2.12 in [JOPP]). In particular, they have discrete spectra.

Denote by $\Delta_{\omega, \mathrm{p}}$ and $\Delta_{\omega_{U} \mid \omega, \mathrm{p}}$ the pure point parts of $\Delta_{\omega}$ and $\Delta_{\omega_{U} \mid \omega}$. Eq. (7.37) implies that

$$
\Delta_{\omega, \mathrm{p}}=\Delta_{\rho} \otimes \Delta_{\nu, \mathrm{p}}, \quad \Delta_{\omega_{U} \mid \omega, \mathrm{p}}=\Delta_{\rho_{U} \mid \rho} \otimes \Delta_{\nu, \mathrm{p}},
$$

where $\Delta_{\nu, \mathrm{p}}$ is the pure point part of $\Delta_{\nu}$. Since $\Delta_{\nu}=\mathrm{e}^{-\beta L_{\mathcal{R}}}$, the operators $\Delta_{\omega, \mathrm{p}}^{\mathrm{i} \alpha}$ and $\Delta_{\omega_{U} \mid \omega, \mathrm{p}}^{\mathrm{i} \alpha}$ are trace class by assumption and it follows from Eq. (7.36) that these two operators are unitarily equivalent so that

$$
\operatorname{tr}\left(\Delta_{\omega, \mathrm{p}}^{\mathrm{i} \alpha}\right)=\operatorname{tr}\left(\Delta_{\omega_{U} \mid \omega, \mathrm{p}}^{\mathrm{i} \alpha}\right)
$$

for all $\alpha \in \mathbb{C}$. Using Eq. (7.38) and (7.39), an explicit calculation yields

$$
\begin{gathered}
\operatorname{tr}\left(\Delta_{\omega, \mathrm{p}}^{\mathrm{i} \alpha}\right)=\operatorname{tr}\left(\Delta_{\rho}^{\mathrm{i} \alpha}\right) \operatorname{tr}\left(\Delta_{\nu, \mathrm{p}}^{\mathrm{i} \alpha}\right)=\operatorname{tr}\left(\rho^{\mathrm{i} \alpha}\right) \operatorname{tr}\left(\rho^{-\mathrm{i} \alpha}\right) \operatorname{tr}\left(\Delta_{\nu, \mathrm{p}}^{\mathrm{i} \alpha}\right), \\
\operatorname{tr}\left(\Delta_{\omega_{U} \mid \omega, \mathrm{p}}^{\mathrm{i} \alpha}\right)=\operatorname{tr}\left(\Delta_{\rho_{U} \mid \rho}^{\mathrm{i} \alpha}\right) \operatorname{tr}\left(\Delta_{\nu, \mathrm{p}}^{\mathrm{i} \alpha}\right)=\operatorname{tr}\left(\rho_{U}^{\mathrm{i} \alpha}\right) \operatorname{tr}\left(\rho^{-\mathrm{i} \alpha}\right) \operatorname{tr}\left(\Delta_{\nu, \mathrm{p}}^{\mathrm{i} \alpha}\right) .
\end{gathered}
$$

Thus, we conclude that

$$
\operatorname{tr}\left(\rho^{\mathrm{i} \alpha}\right)=\operatorname{tr}\left(\rho_{U}^{\mathrm{i} \alpha}\right)
$$

for all $\alpha \in \mathbb{C}$, which implies that $\rho$ and $\rho_{U}$ are unitarily equivalent.

Proof of Proposition 4.1 The proof is based on an application of the real analytic implicit function theorem. Denote by $\mathfrak{X}$ the real vector space $\left\{X \in \mathcal{O}_{\mathcal{S}}^{\text {sa }} \mid \operatorname{tr}(X)=0\right\}$ equipped with the inner product $(X, Y)=\operatorname{tr}(X Y)$. Let

$$
\mathbb{R} \times \mathfrak{X} \ni(\lambda, X) \mapsto F(\lambda, X)=\rho_{X+\lambda V}-\rho_{\mathrm{f}} \in \mathfrak{X}
$$


First, note that $F$ is real analytic. Moreover, for any $X \in \mathfrak{X}$, one has

$$
F(0, X)=\frac{\mathrm{e}^{-\beta X}}{\operatorname{tr}\left(\mathrm{e}^{-\beta X}\right)}-\rho_{\mathrm{f}}
$$

and $F(0, X)=0$ iff $X=H_{0}$. Let $L$ be the standard Liouvillean of the group $\tau_{H_{0}+\lambda V}$ and $\Phi \in \mathcal{P}$ the standard representative of the KMS state $\mu_{H+\lambda V}$. For $X, Y \in \mathfrak{X}$ one has

$$
\left(\rho_{H+X+\lambda V}, Y\right)=\mu_{H+X+\lambda V}(Y \otimes \mathbb{1})=\frac{\left\langle\mathrm{e}^{-\beta(L+\pi(X)) / 2} \Phi, \pi(Y \otimes \mathbb{1}) \mathrm{e}^{-\beta(L+\pi(X)) / 2} \Phi\right\rangle}{\left\|\mathrm{e}^{-\beta(L+\pi(X)) / 2} \Phi\right\|^{2}} .
$$

Using Eq. (7.35), an explicit calculation yields that the derivative $F^{\prime}\left(\lambda, H_{0}\right)$ of the function $F$ with respect to its second argument is the symmetric linear map on $\mathfrak{X}$ given by

$$
\left(F^{\prime}\left(\lambda, H_{0}\right) X, Y\right)=-2 \int_{0}^{\beta / 2} \operatorname{Re}\left\langle\mathrm{e}^{-s L / 2} \pi(\hat{X} \otimes \mathbb{1}) \Psi, \mathrm{e}^{-s L / 2} \pi(\hat{Y} \otimes \mathbb{1}) \Psi\right\rangle \mathrm{d} s,
$$

where $\hat{X}=X-\rho_{H_{0}+\lambda V}(X) \mathbb{1}$. Since $\hat{X}=0$ iff $X=0$, it follows that

$$
\left(F^{\prime}\left(\lambda, H_{0}\right) X, X\right)=-2 \int_{0}^{\beta / 2}\left\|\mathrm{e}^{-s L / 2} \pi(\hat{X} \otimes \mathbb{1}) \Psi\right\|^{2} \mathrm{~d} s<0
$$

for all $0 \neq X \in \mathfrak{X}$, and the implicit function theorem yields the conclusions of Proposition 4.1.

Proof of Proposition 4.2 Suppose that $\sigma(\gamma)=0$. The weak-* lower semicontinuity of relative entropy yields

$$
0=\sigma(\gamma)=\lim _{t \rightarrow \infty} S\left(\omega_{\mathrm{i}} \circ \tau_{K_{\gamma}}^{t} \mid \rho_{K_{\gamma}}(t) \otimes \nu_{\mathrm{i}}\right) \geq S\left(\mu_{K_{\gamma}} \mid \rho_{\mathrm{f}} \otimes \nu_{\mathrm{i}}\right)
$$

which implies $\mu_{K_{\gamma}}=\rho_{\mathrm{f}} \otimes \nu_{\mathrm{i}}$ and hence $\mu_{K_{\gamma}} \circ \tau_{\mathcal{R}}^{t}=\mu_{K_{\gamma}}=\mu_{K_{\gamma}} \circ \tau_{K_{\gamma}}^{t}$ for all $t \in \mathbb{R}$. It follows that

$$
\mu_{K \gamma}\left(\delta_{\mathcal{R}}(A)\right)=0=\mu_{K_{\gamma}}\left(\delta_{\mathcal{R}}(A)+\mathrm{i}\left[K_{\gamma}, A\right]\right)
$$

for all $A \in \operatorname{Dom}\left(\delta_{\mathcal{R}}\right)$, from which we conclude that $K_{\gamma}$ belongs to the centralizer of $\mu_{K_{\gamma}}$. It follows from the KMS property of $\mu_{K_{\gamma}}$ that $\tau_{K_{\gamma}}^{t}\left(K_{\gamma}\right)=K_{\gamma}$ for all $t \in \mathbb{R}$ (see, e.g., Proposition 5.3.28 in [BR2]).

For $\zeta \in \mathbb{R}$, set $S_{\zeta}=\mathrm{e}^{\zeta K_{\gamma}} / \mu_{K_{\gamma}}\left(\mathrm{e}^{2 \zeta K_{\gamma}}\right)^{1 / 2}$ and note that $\xi_{\zeta}(A)=\mu_{K_{\gamma}}\left(S_{\zeta} A S_{\zeta}\right)$ defines a state in $\mathcal{N}_{\omega_{\mathrm{i}}}$. The mixing property and the fact that $\tau_{K_{\gamma}}^{t}\left(S_{\zeta}\right)=S_{\zeta}$ yield

$$
\mu_{K_{\gamma}}(A)=\lim _{t \rightarrow \infty} \xi_{\zeta} \circ \tau_{K_{\gamma}}^{t}(A)=\lim _{t \rightarrow \infty} \mu_{K_{\gamma}}\left(S_{\zeta} \tau_{K_{\gamma}}^{t}(A) S_{\zeta}\right)=\xi_{\zeta}(A),
$$

from which we conclude that $\mu_{K_{\gamma}}\left(S_{\zeta} A S_{\zeta}-A\right)=0$ for all $A \in \mathcal{O}$. Setting $A=S_{\zeta}^{2}-\mathbb{1}$ further yields $\mu_{K_{\gamma}}\left(\left(S_{\zeta}^{2}-\mathbb{1}\right)^{2}\right)=0$. Since $\mu_{K_{\gamma}}$ is faithful we conclude that $S_{\zeta}^{2}=\mathbb{1}$ and hence that $K_{\gamma}$ is a multiple of $\mathbb{1}$. This implies that $\delta_{K_{\gamma}}=\delta_{\mathcal{R}}$ and contradicts Assumption A.

Proof of Theorem 5.1 Denote by $L$ the standard Liouvillean of the group $\tau_{K(0)}$. Let $\Psi(0)$ be the standard vector representative of the KMS state $\mu_{K(0)}$. For $t \in[0, T]$, set

$$
L_{T}(t)=L+\pi\left(\widehat{K}_{T}(t)\right)-J \pi\left(\widehat{K}_{T}(t)\right) J
$$

with $\widehat{K}_{T}(t)=K_{T}(t)-K_{T}(0)$. The family $\left\{W_{T}(t)\right\}_{t \in[0, T]}$ of unitary operators on $\mathfrak{H}$ satisfying

$$
\mathrm{i} \partial_{t} W_{T}(t)=L_{T}(t) W_{T}(t), \quad W_{T}(0)=I,
$$

implements the dynamics $\alpha_{K_{T}}$ and preserves the natural cone, i.e.,

$$
\pi\left(\alpha_{K_{T}}^{t}(A)\right)=W_{T}^{*}(t) \pi(A) W_{T}(t), \quad W_{T}(t) \mathcal{P} \subset \mathcal{P},
$$


for all $t \in[0, T]$ and all $A \in \mathcal{O}$. With $\widehat{K}(\gamma)=K(\gamma)-K(0)$, the standard Liouvillean of the instantaneous dynamics $\tau_{K(\gamma)}$ is

$$
L(\gamma)=L+\pi(\widehat{K}(\gamma))-J \pi(\widehat{K}(\gamma)) J
$$

and the standard representative of the KMS state $\mu_{K(\gamma)}$ is

$$
\Psi(\gamma)=\frac{\mathrm{e}^{-\beta(L+\pi(\widehat{K}(\gamma))) / 2} \Psi(0)}{\left\|\mathrm{e}^{-\beta(L+\pi(\widehat{K}(\gamma))) / 2} \Psi(0)\right\|} .
$$

By construction, the orthogonal projection

$$
P(\gamma)=|\Psi(\gamma)\rangle\langle\Psi(\gamma)|
$$

is such that $\operatorname{Ran}(P(\gamma)) \subset \operatorname{Ker}(L(\gamma))$ for $\gamma \in[0,1]$. Moreover, Assumption $\mathbf{B}$ implies $\operatorname{Ran}(P(\gamma))=$ $\operatorname{Ker}(L(\gamma))$ for $\gamma \in] 0,1[$. Since the function $] 0,1\left[\ni \gamma \mapsto \widehat{K}(\gamma)\right.$ is $C^{2}$ in norm with uniformly bounded first and second derivative, the expansion (7.34), the estimate (7.33), and an obvious telescoping argument show that the map

$$
] 0,1[\ni \gamma \mapsto P(\gamma) \in \mathcal{B}(\mathfrak{H})
$$

is also $C^{2}$ in norm with uniformly bounded first and second derivative.

One easily checks that the adiabatic evolution $\mathcal{W}_{T}(t)$ defined by

$$
\mathrm{i} \partial_{t} \mathcal{W}_{T}(t)=\left(L_{T}(t)+T^{-1} \mathrm{i}[\dot{P}(t / T), P(t / T)]\right) \mathcal{W}_{T}(t), \quad \mathcal{W}_{T}(0)=\mathbb{1} .
$$

intertwines $P(0)$ and $P(t / T)$, i.e., that

$$
\mathcal{W}_{T}(t) P(0)=P(t / T) \mathcal{W}_{T}(t)
$$

holds for $t \in[0, T]$.

With these preliminaries, the Avron-Elgart adiabatic theorem [AE, Teu, ASF1] gives:

Theorem 7.1 Suppose that Assumption B holds. Then

$$
\lim _{T \rightarrow \infty} \sup _{t \in[0, T]}\left\|W_{T}(t)-\mathcal{W}_{T}(t)\right\|=0 .
$$

For $\gamma \in[0,1]$, it follows from Eq. (7.40) that

$$
\mu_{K(0)} \circ \alpha_{K_{T}}^{\gamma T}(A)=\left\langle W_{T}(\gamma T) \Omega, \pi(A) W_{T}(\gamma T) \Omega\right\rangle,
$$

while the intertwining relation (7.41) yields

$$
\mu_{K(\gamma)}(A)=\left\langle\mathcal{W}_{T}(\gamma T) \Omega, \pi(A) \mathcal{W}_{T}(\gamma T) \Omega\right\rangle .
$$

Thus, we have the estimate

$$
\left|\mu_{K(0)} \circ \alpha_{K_{T}}^{\gamma T}(A)-\mu_{K(\gamma)}(A)\right| \leq 2\|\mathrm{~A}\| \sup _{t \in[0, T]}\left\|W_{T}(t)-\mathcal{W}_{T}(t)\right\|,
$$

which, together with Theorem 7.1, yields Theorem 5.1. 
Proof of Proposition 5.2 We use the same notation as in the proof of Theorem 5.1. Set $\Phi(\gamma)=$ $\mathrm{e}^{-\beta(L+\pi(\widehat{K}(\gamma))) / 2} \Psi(0)$. Araki's perturbation formula yields

$$
S\left(\omega \mid \omega_{K(\gamma)}\right)=S\left(\omega \mid \omega_{\mathrm{i}}\right)+\beta \omega(\widehat{K}(\gamma))+\log \|\Phi(\gamma)\|^{2}
$$

for any $\omega \in \mathcal{N}_{\omega_{\mathrm{i}}}$. Setting $\omega=\omega_{\mathrm{i} / \mathrm{f}}$ and $\gamma=0 / 1$ we derive $\|\Phi(0)\|=\|\Phi(1)\|=1$. Next, we claim that

$$
\mu_{K(\gamma)}\left(\partial_{\gamma} K(\gamma)\right)=-\frac{1}{\beta} \partial_{\gamma} \log \|\Phi(\gamma)\|^{2}
$$

which clearly implies Proposition 5.2.

The identity

implies that (7.42) follows from

$$
\partial_{\gamma} \log \|\Phi(\gamma)\|^{2}=\frac{\left\langle\partial_{\gamma} \Phi(\gamma), \Phi(\gamma)\right\rangle+\left\langle\Phi(\gamma), \partial_{\gamma} \Phi(\gamma)\right\rangle}{\|\Phi(\gamma)\|^{2}}
$$

$$
\left\langle\Phi(\gamma), \partial_{\gamma} \Phi(\gamma)\right\rangle=-\frac{\beta}{2}\left\langle\Phi(\gamma), \pi\left(\partial_{\gamma} K(\gamma)\right) \Phi(\gamma)\right\rangle
$$

The last identity is a direct consequence of Eq. (7.35) and the fact that $L(\gamma) \Phi(\gamma)=0$.

\section{References}

[AE] Avron, J., and Elgart, A.: Adiabatic theorem without a gap condition. Commun. Math. Phys. 203, 445-463 (1999).

[AHHH] Alicki, R., Horodecki, M., Horodecki, P., and Horodecki, R.: Thermodynamics of quantum information systems-Hamiltonian description. Open Systems and Information Dynamics 11, 205-217 (2004).

[AJPP1] Aschbacher, W., Jakšić, V., Pautrat, Y., and Pillet, C.-A.: Topics in non-equilibrium quantum statistical mechanics. In Open Quantum Systems III. Recent Developments. S. Attal, A. Joye and C.-A. Pillet editors. Lecture Notes in Mathematics 1882. Springer, Berlin, 2006.

[AJPP2] Aschbacher, W., Jakšić, V., Pautrat, Y., and Pillet, C.-A.: Transport properties of quasi-free Fermions. J. Math. Phys. 48, 032101-1-28 (2007).

[AM] Aizenstadt, V.V., and Malyshev, V.A.: Spin interaction with an ideal Fermi gas. J. Stat. Phys. 48, 51-68, (1987).

[Ar1] Araki, H.: Relative Hamiltonian for faithful normal states of a von Neumann algebra. Publ. Res. Inst. Math. Sci. Kyoto Univ. 9, 165-209 (1973).

[Ar2] Araki, H.: Relative entropy of states of von Neumann algebras. Publ. Res. Inst. Math. Sci. Kyoto Univ. 11, 809-833 (1975/76).

[Ar3] Araki, H.: Relative entropy of states of von Neumann algebras II. Publ. Res. Inst. Math. Sci. Kyoto Univ. 13, 173-192 (1977/78).

[Ar4] Araki, H.: Type of von Neumann algebra associated with free field. Prog. Theor. Phys. 32, 956-965 (1964).

[ArW] Araki, H., and Woods, E.J.: A classification of factors. Publ. Res. Inst. Math. Sci. Kyoto Univ. Ser. A 3, 51-130 (1968).

[AS] Abou Salem, W.K.: On the quasi-static evolution of nonequilibrium steady states. Ann. Henri Poincaré 8, 569-596 (2007). 
[ASF1] Abou Salem, W.K., and Fröhlich, J.: Adiabatic theorems and reversible isothermal processes. Let. Math. Phys, 72, 152-163 (2005).

[ASF2] Abou Salem, W.K., and Fröhlich, J.: Cyclic thermodynamic processes and entropy production. J. Stat. Phys. 126, 431-466 (2007).

[ASF3] Abou Salem, W.K., and Fröhlich, J.: Status of the fundamental laws of thermodynamics. J. Stat. Phys. 126, 1045-1068 (2007).

[ASF4] Abou Salem, W.K., and Fröhlich, J.: Adiabatic theorems for quantum resonances. Commun. Math. Phys. 273, 651-675 (2007).

[Ber] Bérut, A., Arakelyan, A., Petrosyan, A., Ciliberto, S., Dillenscheider, R., and Lutz, E.: Experimental verification of Landauer's principle linking information and thermodynamics. Nature 483, 187-189 (2012).

[BF] Birke, L., and Fröhlich, J.: KMS etc. Rev. Math. Phys. 14, 829-871 (2002).

[BFS] Bach, V., Fröhlich, J., and Sigal, I.M.: Return to equilibrium. J. Math. Phys. 41, 3985-4060, (2000).

[BJM] Bruneau, L., Joye, A., and Merkli, M.: Repeated interactions in open quantum systems. To appear in J. Math. Phys.

[BM] Botvich, D.D., and Malyshev, V.A.: Unitary equivalence of temperature dynamics for ideal and locally perturbed Fermi-gas. Commun. Math. Phys. 91, 301-312 (1983).

[BR1] Bratteli, O., and Robinson, D.W.: Operator Algebras and Quantum Statistical Mechanics I. Second Edition. Springer, Berlin, 1987.

[BR2] Bratteli, O., and Robinson, D.W.: Operator Algebras and Quantum Statistical Mechanics II. Second Edition. Springer, Berlin, 1997.

[CSt] Connes, A., and Størmer, E.: Homogeneity of the state space of factors of type III 1 . J. Funct. Anal. 28, 187-196 (1978).

[Dav] Davies, E.B.: Markovian master equations. Commun. Math. Phys. 39, 91-110 (1974).

[DF] Dereziński, J., and Früboes, R.: Fermi golden rule and open quantum systems. In Open Quantum Systems III. Recent Developments. S. Attal, A. Joye and C.-A. Pillet editors. Lecture Notes in Mathematics 1882, Springer, Berlin, 2006.

[dGM] de Groot, S.R., and Mazur, P.: Nonequilibrium Thermodynamics. North Holland, Amsterdam, 1962.

[DJ] Dereziński, J., and Jakšić, V.: Return to equilibrium for Pauli-Fierz systems. Ann. Henri Poincaré 4, 739-793 (2003).

[DJP] Dereziński, J., Jakšić, V., and Pillet, C.-A.: Perturbation theory of $W^{*}$-dynamics, Liouvilleans and KMS-states. Rev. Math. Phys. 15, 447-489 (2003).

[Don] Donald, M.J.: Relative Hamiltonians which are not bounded from above. J. Funct. Anal. 91, 143 (1990).

[dR] de Roeck, W.: Large deviation generating function for currents in the Pauli-Fierz model. Rev. Math. Phys. 21, 549-585 (2009).

[dRK] de Roeck, W., and Kupianien, A.: Return to equilibrium for weakly coupled quantum systems: A simple polymer expansion. Commun. Math. Phys. 305, 1-30 (2011). 
[DS] Davies, E.B., and Spohn, H.: Open quantum systems with time-dependent Hamiltonians and their linear response. J. Stat. Phys. 19, 511-523 (1978).

[FM] Fröhlich, J., and Merkli, M.: Another return of "return to equilibrium". Commun. Math. Phys. 251, 235-262 (2004).

[FMSU] Fröhlich, J., Merkli, M., Schwarz, S., and Ueltschi, D.: Statistical Mechanics of Thermodynamic Processes. In A garden of quanta. Essays in Honor of Hiroshi Ezawa. J. Arafune, A. Arai, M. Kobayashi, K. Nakamura, T. Nakamura, I. Ojima, N. Sakai, A. Tonomura and K. Watanabe editors. World Scientific Publishing, Singapore, 2003.

[FMU] Fröhlich, J., Merkli, M., and Ueltschi, D.: Dissipative transport: Thermal contacts and tunneling junctions. Ann. Henri Poincaré 4, 897-945 (2003).

[Han] Hanson, E.: Summer research project, McGill University (2014).

[HHW] Haag, R., Hugenholtz, N.M., Winnink, M.: On the equilibrium states in quantum statistical mechanics. Commun. Math. Phys. 5, 215-223 (1967).

[Hu] Hugenholtz, N.M.: On the factor type of equilibrium states in quantum statistical mechanics. Commun. Math. Phys. 6, 189-193 (1967).

[JOP1] Jakšić, V., Ogata, Y., and Pillet, C.-A.: The Green-Kubo formula for the spin-fermion system. Commun. Math. Phys., 268, 369-401 (2006).

[JOP2] Jakšić, V., Ogata, Y., and Pillet, C.-A: The Green-Kubo formula for locally interacting fermionic open systems. Ann. Henri Poincaré 8, 1013-1036 (2007).

[JOPP] Jakšić, V., Ogata, Y., Pautrat, Y., and Pillet, C.-A.: Entropic fluctuations in quantum statistical mechanics - an introduction. In Quantum Theory from Small to Large Scales. J. Fröhlich, M. Salmhofer, V. Mastropietro, W. De Roeck and L.F. Cugliandolo editors. Oxford University Press, Oxford, 2012.

[JOPS] Jaksic, V., Ogata, Y., Pillet, C.-A., and Seiringer, R.: Quantum hypothesis testing and nonequilibrium statistical mechanics. Rev. Math. Phys, 24, 1-67 (2012).

[JP1] Jakšić, V., and Pillet, C.-A.: On a model for quantum friction III. Ergodic properties of the spin-boson system. Commun. Math. Phys. 178, 627-651 (1996).

[JP2] Jakšić, V., and Pillet, C.-A.: Spectral theory of thermal relaxation. J. Math. Phys. 38, 1757-1780 (1997).

[JP3] Jakšić, V., and Pillet C.-A.: On entropy production in quantum statistical mechanics. Commun. Math. Phys. 217, 285-293 (2001).

[JP4] Jakšić, V., and Pillet C.-A.: A note on eigenvalues of Liouvilleans. J. Stat. Phys. 218, 937-941 (2001).

[JP5] Jakšić, V., and Pillet, C.-A.: Mathematical theory of non-equilibrium quantum statistical mechanics. J. Stat. Phys. 108, 787-829 (2002).

[JP6] Jakšić, V., and Pillet, C.-A.: Non-equilibrium steady states of finite quantum systems coupled to thermal reservoirs. Commun. Math. Phys. 226, 131-162 (2002).

[JP7] Jakšić, V., and Pillet, C.-A.: A note on the entropy production formula. Contemp. Math. 327, 175-180 (2003).

[JP8] Jakšić, V., and Pillet, C.-A.: Adiabatic theorem for KMS states. Unpublished.

[JPW] Jakšić, V., Pillet, C.-A, and Westrich, M.: Entropic fluctuations of quantum dynamical semigroups. J. Stat. Phys. 154, 153-187 (2014). 
[Ko] Kosaki, H.: Interpolation theory and the Wigner-Yanase-Dyson-Lieb concavity. Commun. Math. Phys. 87, 315-329 (1982).

[KR] Kadison, R.V., and Ringrose, J.R.: Fundamentals of the Theory of Operator Algebras. Volume I. Elementary Theory. Academic Press, New York, 1983.

[La] Landauer, R.: Irreversibility and heat generation in the computing process. IBM Journal of Research and Development 5, 183-191 (1961).

[LeSp] Lebowitz, J.L., and Spohn, H.: Irreversible thermodynamics for quantum systems weakly coupled to thermal reservoirs. Adv. Chem. Phys. 38, 109-142 (1978).

[Ma] Maroney, O.: Information processing and thermodynamic entropy. In The Stanford Encyclopedia of Philosophy, Fall 2009 Edition. Edward N. Zalta editor. http://plato.stanford.edu/archives/fall2009/entries/information-entropy/.

[McL] McLennan, J.A. Jr.: The formal statistical theory of transport processes. In Advances in Chemical Physics, Volume 5. I. Prigogine editor. Wiley, Hoboken, NJ, 1963.

[MMS1] Merkli, M., Mück, M., and Sigal, I.M.: Instability of equilibrium states for coupled heat reservoirs at different temperatures J. Funct. Anal. 243, 87-120 (2007).

[MMS2] Merkli, M., Mück, M., and Sigal, I.M.: Theory of non-equilibrium stationary states as a theory of resonances. Ann. Henri Poincaré 8, 1539-1593, (2007).

[NT] Narnhofer, H., and Thirring, W.: Adiabatic theorem in quantum statistical mechanics. Phys. Rev. A 26, 3646-3652 (1982).

[O1] Ojima, I.: Entropy production and non-equilibrium stationarity in quantum dynamical systems: physical meaning of Van Hove limit. J. Stat. Phys. 56, 203-226 (1989).

[O2] Ojima, I.: Entropy production and non-equilibrium stationarity in quantum dynamical systems. In Quantum Aspects of Optical Communications. C. Bendjaballah, O. Hirota and S. Reynaud editors. Lecture Notes in Physics 378. Springer, Berlin, 1991.

[OHI] Ojima, I., Hasegawa, H., and Ichiyanagi, M.: Entropy production and its positivity in nonlinear response theory of quantum dynamical systems. J. Stat. Phys. 50, 633-655 (1988).

[OP] Ohya, M., and Petz, D.: Quantum Entropy and Its Use. Second edition. Springer, Heidelberg, (2004).

[Pi1] Pillet, C.-A.: Entropy production in classical and quantum systems. Markov Proc. Relat. Fields 7, 145-157, (2001).

[Pi2] Pillet, C.-A.: Quantum dynamical systems. In Open Quantum Systems I. The Hamiltonian Approach. S. Attal, A. Joye and C.-A. Pillet editors. Lecture Notes in Mathematics 1880. Springer, Berlin, 2006.

[PW1] Pusz W., and Woronowicz, S.L.: Passive states and KMS states for general quantum systems. Commun. Math. Phys. 58, 273-290 (1978).

[PW2] Pusz, W., and Woronowicz, S.L.: Form convex functions and the WYDL and other inequalities. Lett. Math. Phys. 2, 505-512 (1978).

[Raq] Raquépas, R.: Summer research project, McGill University (2014).

[ReWo] Reeb, D., and Wolf M.M.: (Im-)Proving Landauer's Principle. Preprint arXiv:1306.4352v2 (2013).

[Rob] Robinson, D.W.: Return to equilibrium. Commun. Math. Phys. 31, 171-189, (1973). 
[Ru1] Ruelle, D.: Natural nonequilibrium states in quantum statistical mechanics. J. Stat. Phys. 98, 57-75 (2000).

[Ru2] Ruelle, D.: Entropy production in quantum spin systems. Commun. Math. Phys. 224, 3-16 (2001).

[Si] Simon, B.: The Statistical Mechanics of Lattice Gases I. Princeton University Press, Princeton, NJ, 1993.

[Sp1] Spohn, H.: An algebraic condition for the approach to equilibrium of an open N-level system. Lett. Math. Phys. 2, 33-38 (1977).

[Sp2] Spohn, H.: Entropy production for quantum dynamical semigroups. J. Math. Phys. 19, 12271230 (1978).

[Teu] Teufel, S.: A note on the adiabatic theorem without gap condition. Lett. Math. Phys., 58, 261266 (2001).

[Th] Thirring, W.: Quantum Mathematical Physics: Atoms, Molecules and Large Systems. Second edition, Springer, Berlin, 2002.

[TM] Tasaki, S., and Matsui, T.: Fluctuation theorem, non-equilibrium steady states and MacLennanZubarev ensembles of a class of large systems. In Fundamental Aspects of Quantum Physics, L. Accardi and S. Tasaki editors. QP-PQ: Quantum Probability and White Noise Analysis 17. World Scientific Publishing, Singapore, 2003.

[Zu1] Zubarev, D.N.: The statistical operator for nonequilibrium systems. Sov. Phys. Dokl. 6, 776-778 (1962).

[Zu2] Zubarev, D.N.: Nonequilibrium Statistical Thermodynamics. Consultants, New York (1974). 